ICRR-Report-301-93-13 August, 1993

\title{
Reheating during Hierarchical Clustering in the Universe Dominated by the Cold Dark Matter
}

\author{
M. Fukugita \\ Yukawa Institute for Theoretical Physics, Kyoto University, Kyoto 606 \\ and \\ M. Kawasaki \\ Institute for Cosmic Ray Research, University of Tokyo, Tanashi, Tokyo 188
}

\begin{abstract}
We investigate reheating of the universe by early formation of stars and quasars in the hierarchical clustering scheme of cold dark matter scenario, with perturbation fluctuations normalized by the COBE data. It is found that ionizing uv flux from OB stars with the abundance given by the standard initial mass function is strong enough to ionize the universe from $z \approx 30$ to the present epoch, if $1-2 \%$ of the collapsed baryons go into stars. This lessens significantly the CMB anisotropies at a small angular scale. Reionization also increases the Jeans mass to $M_{\text {luminous }} \approx 10^{9} M_{\odot}$ for $z \lesssim 10$, which leads to a cut-off of the luminosity function of normal galaxies on a faint side. A strong uv flux is expected at $z \approx 2-5$, and the null result of the GunnPeterson test is naturally explained. Early star formation also results in production of heavy elements, and the observational metal abundance sets a strong constraint on the photon energy injection into the intergalactic space.
\end{abstract}

Key words: cosmology:theory - dark matter - diffuse radiation - galaxies:formation 


\section{Introduction}

The currently most attractive scenario for cosmic structure formation assumes the cold dark matter dominated universe and the initial fluctuations originating from adiabatic Gaussian perturbations with scale-invariant spectrum (Peebles 1982, Blumenthal et al.1984). The fluctuations enter non-linear regime at redshift $z \lesssim 50$, depending upon the mass of the fluctuations and the magnitude of each peak of the Gaussian noise, and make gravitationally bound objects. A number of N-body simulations have shown that this is indeed a successful scenario for formation of large scale structure (e.g., Frenk et al. 1988) and also of galaxies (White \& Frenk 1992). The evidence in support for this scenario has been given by the COBE-DMR experiment for anisotropies of cosmic microwave background radiation (CMB); the data show that the fluctuations just after the epoch of recombination for angular scales larger than a few degree are close to scale-invariant spectrum with the magnitude almost correctly given by the CDM model of structure formation (Smoot et al. 1992). It is also pointed out that the CDM dominated universe predicts correctly the epoch and the abundance of bright quasars with the assumption that they were formed from high standard deviation peaks of the fluctuations (Efstathiou \& Rees 1988).

At a more quantitative level the standard CDM model does not quite fit the precise perturbation spectrum given by COBE-DMR and that from correlation functions of galaxies (Efstathiou, Bond \& White 1992; Davis, Summers \& Schlegel 1992), which motivates some authors to consider modifications of the model (Adams et al. 1992; Davis, Summers \& Schlegel 1992; Klypin et al. 1992; Efstathiou, Bond \& White 1992). The original model, however, seems to be at least a good enough starting ground for a consideration of the history of the universe.

In the CDM scenario structure formation is hierarchical. A small object becomes a gravitationally bound system first, and the mass of the bound systems gradually increases by merging. In this scenario formation of objects with a specific mass is not instantaneous, but statistical, and the abundance of bound objects with a specific 
mass varies as a function of redshift.

When bound systems are formed, it is likely that a substantial fraction of baryons form stars, if the cooling time is shorter than the dynamical time. The photons emitted from OB stars would then ionize the universe, which would affect formation and evolution of cosmic structure, as first pointed out by Doroshkevich, Zeldovich and Novikov (1967) and then discussed by Couchman and Rees (1986). In this paper we examine detailed thermal history of the universe in the epoch at $z<100$ taking account of the effect of star formation by explicitly solving the evolution equation for thermal history, and discuss the consequences on formation of galaxies and background photons in the universe. We also take into account the uv flux from early quasars expected in the CDM model.

Another interesting consequence of early star formation is that it results in production of heavy elements, as needed from the metal observation of globular clusters. Actually the observed metal abundance in population II objects sets a strong constraint on the fraction of baryons which go into to stars, and hence on the energy injected into the intergalactic space.

In our work we take the Press-Schechter theory (Press \& Schechter 1974) for hierarchical clustering, since it is known to describe well the clustering observed in Nbody simulations (e.g., Efstathiou \& Rees 1988). We fix the normalization of the Gaussian field by the data given from the COBE-DMR experiment. We confine ourselves to a flat universe with $\Omega=1$ for simplicity. We assume that a certain fraction of collapsed baryons become stars, in so far as the cooling condition is satisfied. We also assume the initial mass function as observed in the Milky Way today (Shapiro \& Teukolsky 1983), and adopt blackbody spectrum with temperature specified by the temperature-mass relation for population II stars. Quasar formation is normalized to the observed abundance at $z=2$. The only free parameters of our calculation is the fraction of baryons which form stars apart from the Hubble constant.

The aspects which we pay a particular attention in the present work are as follows: 
Whether the universe is reionized and whether it recombines again at low redshift is of our prime interest. If the universe would be reionized early enough comparable to a unit Thomson optical depth, this lessens anisotropies of CMB at an angular scale smaller than a degree, which would make them consistent with the limit from the South Pole II experiment (Gaier et al. 1992). Ionization of the universe would increase the Jeans mass, which otherwise is very small compared with the galaxy mass today, to the order of irregular galaxies. The exact Jeans mass limit depends on how efficient is ionization. The evolution of residual photons is also of significant interest. For $z \lesssim 5$ the Gunn-Peterson test (Gunn \& Peterson 1965; Steidel \& Sargent 1986; Jenkins \& Ostriker 1991; Webb et al. 1992) requires that the universe be ionized to a high degree. This means that a reasonable amount of the uv flux must exist all the time for the relevant redshift range to keep the universe ionized. The necessity of uv flux at around $z \approx 4-2$ is also motivated by the presence of Lyman $\alpha$ clouds; the uv flux heats up the clouds so that they do not collapse (Sargent et al. 1980; Ostriker \& Ikeuchi 1983; Rees 1986).

The effect of reheating has been studied by a number of authors (Couchman 1985, Stebbins \& Silk 1986; Couchman \& Rees 1986, Bartlett \& Stebbins 1991; Barcons et al. 1991; Fukugita \& Kawasaki 1990; 1993; Gnedin \& Ostriker 1992; see Cen et al 1990; Cen \& Ostriker 1992 for a different class of the reheating model) in different contexts and models. In particular Couchman \& Rees (1986) studied qualitatively the possibility of reheating in the hierarchical clustering scenario. We shall study more quantitatively the thermal history of the universe, with the strength of the fluctuations determined from the CMB anisotropy observations. Reheating in the hierarchical clustering was also considered by Sasaki et al. (1993) However, these authors take account only of the energy liberated from the gravitationally binding objects. Such scenario basically does not ionize the CDM universe. The energy injection from stars is crucial for reionization.

In sect. 2 the basic model of the present calculation is described. The method 
of the calculation for the thermal history is given in sect.3; the thermal processes that we considered are described in detail. In sect. 4 we present the results of our calculation and discuss the meaning and implications of the results. Sect.5 is given to a conclusion.

\section{The model}

\subsection{Press-Schechter theory}

In the Press-Schechter theory the comoving number density of non-linear objects within the mass range between $M$ and $M+d M$ is given by

$$
N(z, M) d M=\sqrt{\frac{2}{\pi}} \frac{\rho_{0}}{M} \frac{\delta_{c}}{D_{1}(z)}\left(-\frac{1}{\sigma^{2}(M)} \frac{\partial \sigma}{\partial M}\right) \exp \left(-\frac{\delta_{c}^{2}}{2 \sigma^{2}(M) D_{1}^{2}(z)}\right),
$$

where $\rho_{0}$ is the mean comoving mass density, $\delta_{c}$ is the overdensity threshold for the collapse $\left(=1.68\right.$ corresponding to the prediction of spherical collapse model), $D_{1}(Z)$ is the Peebles function for the growth of the perturbations $\left(D_{1}(z)=(1+z)^{-1}\right.$ for $\Omega=1)$, and $\sigma^{2}(M)$ is the Gaussian variance given by

$$
\sigma^{2}(M)=\frac{1}{2 \pi^{2}} \int_{0}^{\infty} P(k) \exp \left(-r_{M}^{2} k^{2}\right) k^{2} d k
$$

with $r_{M}=\left(M / \rho_{0}\right)^{1 / 3} / \sqrt{2 \pi}$ the width of the Gaussian filter and $P(k)$ the power spectrum of density fluctuations. We use the fitting formula for the CDM powerspectrum given by Bardeen, Bond, Kaiser \& Szalay (1986):

$$
\begin{aligned}
P(k)= & A|T(k)|^{2} k \\
T(k) & =\frac{\ln (1+2.34 q)}{2.34 q} \\
& \times\left[1+3.89 q+(16.1 q)^{2}+(5.46 q)^{3}+(6.71 q)^{4}\right]^{-1 / 4}, \\
q & \equiv \frac{k}{\Omega h^{2} \mathrm{Mpc}^{-1}},
\end{aligned}
$$

where $h$ is the Hubble constant $H_{0}$ in units of $100 \mathrm{~km} \mathrm{sec}^{-1} \mathrm{Mpc}^{-1}$ ). This gives a spectrum quite similar to that given in Efstathiou, Bond \& White (1992), but with a 
slightly more power for small scales. The normalization $A$ of the spectrum is fixed by the COBE-DMR data using the relation (Efstathiou, Bond \& White 1992):

$$
\frac{Q_{\mathrm{rms}}}{T_{0}}=\left(\frac{5}{6 \pi^{2}}\right)^{1 / 2}\left(\frac{H_{0}}{2 c}\right)^{2} \Omega^{0.77} A^{1 / 2},
$$

where $T_{0}$ is the present temperature of $\mathrm{CBR}$ and $Q_{\mathrm{rms}}$ is the fluctuation amplitude at $\sim 10^{\circ}$ (Smoot et al. 1992):

$$
Q_{\mathrm{rms}}=16.7 \pm 4.6 \mu \mathrm{K}
$$

This normalization fixes $N(M, z)$ now uniquely.

The fraction $\Omega_{\text {coll }}$ of the total mass of the universe which collapses into the bound object with mass greater than $M$ is shown in Fig.1(a). The effect of reionization is not taken into account here. The Jeans mass before reionization is given by (Bond \& Szalay 1983)

$$
M_{B, J}=1.4 \times 10^{5} M_{\odot} \Omega_{B} \Omega^{-3 / 2} h^{-1}(1+100 / z)^{-3 / 2}
$$

which stays at a constant value $1.4 \times 10^{4} \Omega^{-3 / 2} M_{\odot}$ for $z \gtrsim 100$ due to residual ionization left over the recombination epoch. The total mass corresponding to this Jeans mass is $3 \times 10^{5} \Omega^{-1 / 2} M_{\odot}$ (or less for a smaller $z$ ).

Another necessary condition for continuing collapse is that the cooling time is shorter than the dynamical time (Blumenthal et al. 1984),

$$
M_{B, c}>3.7 \times 10^{5}\left(\Omega h^{2}\right)^{-0.917}\left(\frac{Y_{e}}{10^{-4}}\right)^{-0.625}\left(\frac{\Omega_{B}}{\Omega}\right)^{-1.04}\left(\frac{1+z}{10}\right)^{-2.75} M_{\odot}
$$

where $Y_{e} \simeq 10^{-5} \Omega_{B}^{-1} \Omega^{1 / 2} h^{-1}$. At $z=50$ the limiting mass is $M \sim 10^{6} M_{\odot}$ and decreases as a function of redshift. Therefore we conclude that the first objects which collapse around $z \approx 50$ should have a mass of $10^{6} M_{\odot}$.

In the following calculation we take account of the effect of reionization on the Jeans mass, which is written

$$
M_{B, J}=1.4 \times 10^{5} M_{\odot} \Omega_{B} \Omega^{-3 / 2} h^{-1}\left(\frac{T_{e}}{\mu T_{\gamma}}\right)^{3 / 2}
$$


where $T_{e}$ is the electron temperature, $T_{\gamma}$ is the temperature of the cosmic background radiation and $\mu$ is the mean molecular weight. When the universe is reheated, $T_{e}$ becomes much larger than $T_{\gamma}$ and $Y_{e}$ increases. Therefore, Jeans mass $M_{B, J}$ increases whereas $M_{B, c}$ decreases. We take the object with $\max \left[\left(\Omega / \Omega_{B}\right) M_{B, c},\left(\Omega / \Omega_{B}\right) M_{B, J}\right]<$ $M<10^{13} M_{\odot}$ as being collapsed. For $M>10^{13} M_{\odot}$ the cooling time becomes longer than the Hubble time again, and galaxies are not formed (Blumenthal et al. 1984).

\subsection{Early formation of stars}

When bound objects are formed, we assume that a constant fraction $f$ of the baryonic gas goes into stars in so far as mass of the objects is greater than the Jeans mass and smaller than the maximal mass of galaxies $\left(=10^{13} M_{\odot}\right)$, and the cooling time is shorter than the dynamical time. We set $f=0$ otherwise. We take the baryon density to be $\Omega_{B}=0.05 h^{-2}$ and $\Omega_{B} / \Omega$ to be universal. We also assume that the mass spectrum of stars is proportional to the present-day initial mass function (IMF), $\xi_{\text {imf }}$, for which we adopt the formula given by Shapiro \& Teukolsky (1983). We take the temperaturemass relation for population II stars (e.g., Bond, Carr \& Hogan 1986):

$$
T_{\mathrm{s}}\left(M_{\mathrm{s}}\right)=6 \times 10^{4} \mathrm{~K} \min \left[\left(\frac{M_{\mathrm{s}}}{100 M_{\odot}}\right)^{0.3}, 1\right] .
$$

Stars with mass smaller than $10 M_{\odot}$ produce very few ionizing photons. Therefore it is sufficient if we only consider the uv flux from stars whose mass is greater than $10 M_{\odot}$. A star with mass $M_{\mathrm{s}}$ produces the radiation energy $\epsilon_{\mathrm{s}} M_{s}$ in the main-sequence time $t_{\mathrm{MS}}$, where $\epsilon_{\mathrm{s}}$ and $t_{\mathrm{MS}}$ are given by

$$
\epsilon_{\mathrm{s}}=0.0046\left(\frac{X}{0.76}\right) \min \left[\left(\frac{M_{\mathrm{s}}}{100 M_{\odot}}\right)^{1 / 2}, 1\right],
$$

and

$$
t_{\mathrm{MS}}=2.3 \times 10^{6} \operatorname{year}\left(\frac{\epsilon_{\mathrm{s}}}{0.0046}\right) \max \left[1,\left(\frac{M_{\mathrm{s}}}{100 M_{\odot}}\right)^{-2}\right],
$$

where $X=0.76$ is the hydrogen mass fraction. Since the main-sequence time of massive stars with $M_{s}>10 M_{\odot}$ is shorter than the cosmic time for $z<50$, we may 
assume that the uv photons are produced instantaneously after the formation of the stars. The production rate of uv photons is then

$$
\left(\frac{d n_{\gamma}\left(\epsilon_{\gamma}\right)}{d z}\right)_{\mathrm{star}}=\int d M_{\mathrm{s}} \frac{B\left(\epsilon_{\gamma}, T_{\mathrm{s}}\right)}{\epsilon_{\gamma}} \epsilon_{\mathrm{s}} \xi_{\mathrm{imf}}\left(M_{\mathrm{s}}\right) f \frac{\Omega_{B}}{\Omega} \int d M M\left(\frac{\partial N(M, z)}{\partial z}\right),
$$

where $B\left(\epsilon_{\gamma}, T_{\mathrm{s}}\right)$ is the blackbody spectrum normalized to $\int d \epsilon_{\gamma} B\left(\epsilon_{\gamma}\right)=1$.

Stars with mass greater than $4 M_{\odot}$ eject heavy elements. Following Carr, Bond \& Arnett (1984) we assume that the fraction of mass ejected as heavy elements is

$$
\begin{array}{ll}
Z_{\mathrm{ej}}=0.5-\left(\frac{M_{\mathrm{s}}}{6.3 M_{\odot}}\right)^{-1} & \text { for } 15<M / M_{\odot}<100 \\
Z_{\mathrm{ej}}=0.1 & \text { for } 8<M / M_{\odot}<15 \\
Z_{\mathrm{ej}}=0.2 & \text { for } 4<M / M_{\odot}<8 .
\end{array}
$$

Then evolution of the metallicity $Z$ is given by

$$
\frac{d Z}{d z}=\int d M_{\mathrm{s}} Z_{\mathrm{ej}} \epsilon_{\mathrm{s}} \xi_{\mathrm{imf}}\left(M_{\mathrm{s}}\right) f \frac{\Omega_{B}}{\Omega} \int d M M\left(\frac{\partial N(M, z)}{\partial z}\right)
$$

In our work we take $f=0.02$ which, as we find, is about the upper limit from the condition that metallicity of population II stars does not exceed $10^{-3}$.

We show in Fig.1 (b) the mass fraction of stars both with and without taking the effect of reionization into account. The figure indicates that a half of the stars are formed before $z=10$. This is because we assumed that stars are made always at a constant fraction of collapsed baryons with the cut-off at $M \leq 10^{13} M_{\odot}$. In reality we should have more stars formed stationally from the collapsed gas, and expect that our approximation would not be good at low redshift, after galaxies are formed to their present form. Fig.1(c) shows the evolution of the emitted radiation energy from stars. In principle, our scheme has a double counting problem in that the baryons which have gone into stars when a small object collapses might be used again to make another stars when a larger object is formed. Since the fraction which goes into stars are very small $\left(\sim 10^{-2}\right)$, however, the double counting effect is actually negligible. 


\subsection{Formation of quasars}

We take account of the effect of quasars following Efstathiou \& Rees (1988). Using the Press-Schechter theory, we estimate the comoving number density of luminous quasars as

$$
\begin{aligned}
N_{\text {quasar }}(z) & =\int_{\max \left[t-t_{\mathrm{Q}}, 0\right]}^{t} \int_{M_{\min }}\left(\frac{\partial N(M, z)}{\partial t}\right) d M d t \\
& \simeq t_{\mathrm{Q}} \int_{M_{\min }}\left(\frac{\partial N(M, z)}{\partial t}\right) d M
\end{aligned}
$$

where $t_{\mathrm{Q}}$ is the lifetime of quasars which is assumed to be much shorter than cosmic time $t$, and $M_{\min }$ is the minimum mass of quasars. We take $L_{\mathrm{Q}, \min }=10^{47} \mathrm{erg} \mathrm{sec}^{-1}$ as a cut-off luminosity of quasars and estimate $M_{\min }$ from the mass-luminosity relation:

$$
M\left(L_{\mathrm{Q}}\right)=2 \times 10^{13} M_{\odot}\left(\frac{t_{\mathrm{Q}}}{10^{8} \text { year }}\right)\left(\frac{\epsilon_{\mathrm{Q}}}{0.1}\right)^{-1}\left(\frac{F_{\mathrm{Q}}}{10^{-4}}\right)^{-1}\left(\frac{L_{\mathrm{Q}}}{10^{47} \mathrm{erg}}\right),
$$

where $F_{\mathrm{Q}}$ is the fraction of the collapsed matter which becomes quasars, and $\epsilon_{\mathrm{Q}}$ is the efficiency defined as the fraction of the rest mass energy that is converted into radiation. The quasar number density depends on $t_{Q}$ and $\epsilon_{\mathrm{Q}} F_{\mathrm{Q}}$. We take $t_{\mathrm{Q}}=10^{8}$ year and fix $\epsilon_{\mathrm{Q}} F_{\mathrm{Q}}$ to be $10^{-5}$ to give the observed comoving number density of quasars brighter than $L_{\mathrm{Q}}=2.5 \times 10^{46} h^{-2} \mathrm{erg} \mathrm{sec}^{-1}$ at $z=2$,

$$
N_{\text {quasar }} \simeq 1.5 \times 10^{-8} h^{-3} \mathrm{Mpc}^{-3}
$$

The uv photon production rate is given by

$$
\left(\frac{d n_{\gamma}\left(\epsilon_{\gamma}, z\right)}{d t}\right)_{\text {quasar }}=\frac{S_{\mathrm{Q}}\left(\epsilon_{\gamma}\right)}{\epsilon_{\gamma}} \int t_{\mathrm{Q}} L_{\mathrm{Q}}\left(\frac{\partial N(M, z)}{\partial t}\right) d M,
$$

where $S_{\mathrm{Q}}\left(\epsilon_{\gamma}\right)$ is the spectrum of the emitted photons which we take to obey a powerlaw of the form (Miralda-Escudé \& Ostriker 1990; model QS2)

$$
S_{\mathrm{Q}} \propto\left\{\begin{array}{cc}
\epsilon_{\gamma}^{-0.4} & \left(\epsilon_{\gamma}<10.2 \mathrm{eV}\right) \\
\epsilon_{\gamma}^{-1.4} & \left(\epsilon_{\gamma}>10.2 \mathrm{eV}\right)
\end{array}\right.
$$

We normalize $S_{\mathrm{Q}}$ as $\int d \epsilon_{\gamma} S_{\mathrm{Q}}=1$. 
The abundance of quasars are given in Fig.1 (d). The first quasars are formed around $z \approx 7-8$. The comoving number density reaches maximum at $z \approx 2.5$ and then it declines. The radiation energy from quasars is shown in Fig.1(c).

\subsection{Heating by collapsing bound objects}

The possibility was discussed that liberation of kinetic energy by radiation cooling during the collapse of galaxies might ionize the universe (Hogan 1980). If free-free cooling or recombination cooling is the dominant cooling process, the produced photons might have energy high enough to ionize the intergalactic medium. This possibility was reconsidered within the context of hierarchical clustering theory recently by Sasaki et al. (1993). We can show, however, that this scenario does not work for the following reasons: for this scenario to work, free-free or recombination cooling (we denote the cooling times as $\tau_{\mathrm{ff}}$ and $\left.\tau_{\text {rec }}\right)$ must be faster than Compton cooling $\left(\tau_{\mathrm{C}}\right)$, expansion cooling $\left(\tau_{H}=1 / H\right)$ and line cooling $\left(\tau_{\text {line }}\right)$ that follows collisional excitation. The conditions $\left(\tau_{\text {ff }}\right.$ or $\left.\tau_{\text {rec }}\right)<\left(\tau_{\mathrm{C}}\right.$ and $\left.\tau_{\mathrm{H}}\right)$ are satisfied only for low temperature, $T<2.5 \times 10^{5} \mathrm{~K}$, as seen in Fig. 2 where the critical temperature for $\tau_{\mathrm{C}}=\tau_{\mathrm{ff}}$ etc. are plotted as a function of redshift. On the other hand, we can show that, at such a low temperature, line cooling dominates over recombination and free-free cooling (Fig.2). This means that liberation of kinetic energy by radiation cooling in binding objects is not efficient enough and does not ionize the universe. We give this argument more quantitatively in Appendix A.

The situation gets worse if we include line cooling due to helium. On the other hand, the constraint might be weakened, if the inhomogeneity effect of baryon density in collapsed objects that enhance recombination (free-free) cooling is taken into account, as discussed by Sasaki et al. (1993). In any case, however, only a very narrow range of temperature may be allowed to produce ionizing photons, even if this enhancement is included. The contributions from massive stars and quasars are far more important in reionizing the universe. We conclude that we can neglect the 
production of ionizing uv photons from collapsing objects.

\section{Thermal history}

\subsection{Method of calculation}

The evolution equations are solved numerically for the ionized fraction of hydrogen and helium atoms $n(\mathrm{HII}) / n_{\mathrm{H}}, n(\mathrm{HeII}) / n_{\mathrm{He}}$ and $n(\mathrm{HeIII}) / n_{\mathrm{He}}$, the photon spectrum $n_{\gamma}\left(\epsilon_{\gamma}, t\right)$, and the electron temperature $T_{e}$ with all relevant thermal processes taken into account. The integration is made with the implicit Euler method. The detailed expressions of thermal processes used in our work are given in order in the following subsections.

We determine the spectral distortion of CMB by solving directly the Kompaneets equation (e.g., Fukugita \& Kawasaki 1990). Since reheating occurs at low $z$ and the temperature is not high in the present model, the distortion is described by the Zeldovich-Sunyaev spectrum and its magnitude is characterized well (within the accuracy in solving numerically the Kompaneets equation) in terms of the Compton yc-parameter:

$$
y_{\mathrm{c}}=\int d t \frac{k\left(T_{e}-T_{\gamma}\right)}{m_{e} c^{2}} n_{e} \sigma_{\mathrm{T}} c
$$

\subsection{Ionization of $\mathrm{H}$ and $\mathrm{He}$}

The abundances of HI, HII, HeI, HeII, and HeIII are determined by the balance between the ionizing processes and the recombination processes. We treat a hydrogen atom as a two level system $(1 S, 2 S+2 P)$ plus continuum following Peebles(1968), Matsuda et al. (1971) and Jones \& Wyse (1985); evolution of the HII fraction is determined by

$$
\begin{aligned}
\frac{d}{d t}\left(\frac{n(\mathrm{HII})}{n_{\mathrm{H}}}\right) & =\frac{R_{1 \mathrm{c}} n(\mathrm{HI})}{n_{\mathrm{H}}}-\frac{\alpha_{2, \mathrm{HII}} n_{e}^{2}}{n_{\mathrm{H}}} \\
& +\frac{R_{2 \mathrm{c}} n(\mathrm{HI})}{n_{H}} \frac{K\left(\alpha_{2, \mathrm{HII}} n_{e}^{2}+\Lambda n(\mathrm{HI}) e^{-h \nu_{\alpha} / k T_{\gamma}}\right)+e^{-h \nu_{\alpha} / k T_{\gamma}}}{1+K\left(\alpha_{2, \mathrm{HII}} n_{e}^{2}+R_{2 \mathrm{c}} n(\mathrm{HI})+\Lambda n(\mathrm{HI})\right)}
\end{aligned}
$$


where $n_{\mathrm{H}} \equiv n(\mathrm{HI})+n(\mathrm{HII}), \Lambda\left(=8.227 \mathrm{sec}^{-1}\right)$ is the two-photon decay rate from $2 S, \nu_{\alpha}$ is the Lyman- $\alpha$ frequency, $K=c^{3} /\left(8 \pi \nu_{\alpha}^{3}\right)(a / \dot{a}), \alpha_{2, \mathrm{HII}}$ is the recombination coefficient to $2 S+2 P$ level and $R_{2 \mathrm{c}}\left(R_{1 \mathrm{c}}\right)$ is the ionization coefficient from $2 S+2 P(1 S) . R_{2 \mathrm{c}}$ and $R_{1 \mathrm{c}}$ are given by

$$
\begin{aligned}
& R_{2 \mathrm{c}}=\gamma_{2 \mathrm{c}}+\beta_{2, \mathrm{HI}} n_{e}+\int_{\epsilon_{2, \mathrm{HI}}} c \sigma_{2 \mathrm{f}, \mathrm{HI}} n_{\gamma}\left(\epsilon_{\gamma}\right) d \epsilon_{\gamma}, \\
& R_{1 \mathrm{c}}=\beta_{1, \mathrm{HI}} n_{e}+\int_{\epsilon_{1, \mathrm{HI}}} c \sigma_{1 \mathrm{f}, \mathrm{HI}} n_{\gamma}\left(\epsilon_{\gamma}\right) d \epsilon_{\gamma}
\end{aligned}
$$

where $\beta_{1, \mathrm{HI}}\left(\beta_{2, \mathrm{HI}}\right)$ is the coefficient for collisional ionization from $1 S(2 S+2 P)$ level, $\gamma_{2 \mathrm{f}}=\alpha_{2, \mathrm{HII}}\left(T_{\gamma}\right)\left(2 m_{e} k T_{\gamma}\right)^{3 / 2} e^{-3.4 \mathrm{eV} / T_{\gamma}}$ is the photo-ionization coefficient due to background photons, $\sigma_{1 \mathrm{f}, \mathrm{HI}}\left(\sigma_{2 \mathrm{f}, \mathrm{HI}}\right)$ is the photo-ionization cross section from $1 S(2 S+2 P)$ and $n_{\gamma}\left(\epsilon_{\gamma}\right)$ is the spectrum of uv photons; we take $\epsilon_{1, \mathrm{HI}}=13.6 \mathrm{eV}, \epsilon_{2, \mathrm{HI}}=10.2 \mathrm{eV}$. Explicit expressions for the coefficients used in this work is summarized in Appendix B.

Since helium is a minor component, we may treat it as a one-level system. The time evolution of HI, HII and HIII is then given by

$$
\begin{aligned}
\frac{d}{d t}\left(\frac{n(\mathrm{HeII})}{n_{\mathrm{He}}}\right) & =\frac{n(\mathrm{HeI})}{n_{\mathrm{He}}} \int_{\epsilon_{\mathrm{HeI}}} d \epsilon_{\gamma} \sigma_{\mathrm{bf}, \mathrm{HeI}} c n_{\gamma}\left(\epsilon_{\gamma}\right) \\
& +\beta_{\mathrm{HeI}} n_{e} \frac{n(\mathrm{HeI})}{n_{\mathrm{He}}}-\beta_{\mathrm{HeII}} n_{e} \frac{n(\mathrm{HeII})}{n_{\mathrm{He}}} \\
& -\alpha_{\mathrm{HeII}} n_{e} \frac{n(\mathrm{HeII})}{n_{\mathrm{He}}}+\alpha_{\mathrm{HeIII}} n_{e} \frac{n(\mathrm{HeIII})}{n_{\mathrm{He}}} \\
& -\xi_{\mathrm{HeII}} n_{e} \frac{n(\mathrm{HeII})}{n_{\mathrm{He}}} \\
\frac{d}{d t}\left(\frac{n(\mathrm{HeIII})}{\left.n_{\mathrm{He}}\right)}\right. & =\frac{n(\mathrm{HeII})}{n_{\mathrm{He}}} \int_{\epsilon_{\mathrm{HeII}}} d \epsilon_{\gamma} \sigma_{\mathrm{bf}, \mathrm{HeII}} c n_{\gamma}\left(\epsilon_{\gamma}\right) \\
& +\beta_{\mathrm{HeII}} n_{e} \frac{n(\mathrm{HeII})}{n_{\mathrm{He}}}-\alpha_{\mathrm{HeIII}} n_{e} \frac{n(\mathrm{HeIII})}{n_{\mathrm{He}}}
\end{aligned}
$$

where $n_{\mathrm{He}} \equiv n(\mathrm{HeI})+n(\mathrm{HeII})+n(\mathrm{HeIII}), \beta, \alpha$ and $\xi$ are the coefficients for the collisional ionization, recombination, and dielectronic recombination, respectively; $\sigma_{\mathrm{bf}}$ 
is the photoelectric ionization cross section, $\epsilon_{\mathrm{HeI}}\left(\epsilon_{\mathrm{HeII}}\right)$ is the ionization energy of HeI (HeII) (see Appendix B).

\section{3 uv spectrum}

The time evolution of the photon spectrum is determined by solving

$$
\begin{aligned}
\frac{d n_{\gamma}\left(\epsilon_{\gamma}\right)}{d t} & =\frac{\dot{a}}{a}\left(\epsilon_{\gamma} \frac{\partial n_{\gamma}}{\partial \epsilon_{\gamma}}-2 n_{\gamma}\right) \\
& +\frac{1}{\epsilon_{\gamma}}\left(j_{\mathrm{ff}}+j_{\mathrm{fb}, \mathrm{HII}}+j_{\mathrm{fb}, \mathrm{HeII}}+j_{\mathrm{fb}, \mathrm{HeIII}}\right) \\
& -\left(\sigma_{1 \mathrm{f}, \mathrm{HI}}+\sigma_{2 \mathrm{f}, \mathrm{HI}}+\sigma_{\mathrm{bf}, \mathrm{HeI}}+\sigma_{\mathrm{bf}, \mathrm{HeII}}\right) c n_{\gamma} \\
& +\left(\frac{d n_{\gamma}}{d t}\right)_{\mathrm{star}}+\left(\frac{d n_{\gamma}}{d t}\right)_{\text {quasar }},
\end{aligned}
$$

where $j_{\mathrm{ff}}$ is the emissivity of the free-free process, $j_{\mathrm{fb}, \mathrm{HII}}, j_{\mathrm{fb}, \mathrm{HeII}}$ and $j_{\mathrm{fb}, \mathrm{HeIII}}$ are that of the free-bound process due to HII, HeII and HeIII, respectively. In the present consideration the electron temperature is relatively low $\left(\lesssim 10^{4} \mathrm{~K}\right)$, and hence the uv photon production due to the free-free process is not important.

The photons produced by recombination process have a complicated spectrum. We treat HI and HeII as two level systems $(1 S, 2 S+2 P)$ and HeI as a three level system $\left(1^{1} S, 2^{1} S, 2^{3} P\right)$. When a free electron is captured by HII, HeII or HeIII, the emmisivity is given by

$$
j_{\mathrm{fb}, i, j}=\frac{\epsilon_{\gamma}}{k T_{e}} \alpha_{i, j} n(i) n_{e} \exp \left(-\left(\epsilon_{\gamma}-\epsilon_{i, j}\right) / k T_{e}\right),
$$

where $i=$ HII, HeII and HeIII, and $j$ represents the energy level; $\alpha_{i, j}$ is the recombination coefficient and $\epsilon_{i, j}$ is the binding energy of the $j$-level of an $i$-atom. Unless an electron recombines directly into the $1 S$ level, a monochromatic (line) photon or two photons are subsequently emitted from an excited atom at every recombination process. The photon emissivity is

$$
j_{\mathrm{fb}, i, j, \text { line }}=\epsilon_{\gamma} \alpha_{i, j} n(i) n_{e} \delta\left[\epsilon_{\gamma}-\left(\epsilon_{i}-\epsilon_{i, j}\right)\right]
$$


for a monochromatic photon emission, and

$$
j_{\mathrm{fb}, i, j, \mathrm{two}}=2 \epsilon_{\gamma} \alpha_{i, j} n(i) n_{e} \delta\left[\epsilon_{\gamma}-\left(\epsilon_{i}-\epsilon_{i, j}\right) / 2\right],
$$

for the two-photon process.円

In case of dielectric recombination of HeII, two electrons are in excited states after recombination, and they go down to the ground state by emitting photons. We treat this process by assuming that the first electron goes to the ground state emitting a photon with energy corresponding to $(2 S+2 P)$ level of HeIII and the second electron emits a photon with energy corresponding to $2^{1} P$ level of HeII. Therefore,

$$
j_{\mathrm{fb}, \mathrm{HeII}, \mathrm{d}}=\epsilon_{\gamma} \xi_{\mathrm{HeII}} n(\mathrm{HeII}) n_{e}\left[\delta\left(\epsilon_{\gamma}-40.81 \mathrm{eV}\right)+\delta\left(\epsilon_{\gamma}-21.2 \mathrm{eV}\right)\right]
$$

\subsection{Electron temperature}

Evolution of the electron temperature is given by

$$
\begin{aligned}
\frac{3}{2} \frac{d}{d t}\left(\frac{k T_{e} n_{B}}{\mu}\right) & =\sum_{i=\mathrm{HI}, \mathrm{HeI}, \mathrm{HeII}} n(i) c \int\left(\epsilon_{\gamma}-\epsilon_{i}\right) n_{\gamma} \sigma_{\mathrm{bf}, i} d \epsilon_{\gamma} \\
& -\sum_{i=\mathrm{HI}, \mathrm{HeI}, \mathrm{HeII}} \zeta_{i} n_{e} n(i) \\
& -\sum_{i=\mathrm{HII}, \mathrm{HeII}, \mathrm{HeIII}} \eta_{i} n_{e} n(i) \\
& -\omega_{\mathrm{HeII}} n_{e} n(\mathrm{HeIII}) \\
& -\sum_{i=\mathrm{HI}, \mathrm{HeI}, \mathrm{HeII}} \psi_{i} n_{e} n(i) \\
& -\lambda_{\mathrm{c}} \\
& -\theta_{\mathrm{ff}}[n(\mathrm{HII})+n(\mathrm{HeII})+4 n(\mathrm{HeIII})] n_{e} \\
& -\frac{15}{2} \frac{\dot{a}}{a}\left(\frac{k T_{e} n_{B}}{\mu}\right)
\end{aligned}
$$

where $\zeta_{i}$ is the collisional-ionization cooling coefficient due to atomic state $i, \eta_{i}$ is the recombination cooling coefficient, $\omega_{\mathrm{HeII}}$ is the dielectronic recombination cooling

\footnotetext{
${ }^{1}$ Here we neglect the effect of the energy distribution for the two-photon process.
} 
coefficient due to HeII, $\psi_{i}$ is the collisional excitation cooling coefficient due to $i, \lambda_{\mathrm{c}}$ is the Compton cooling rate and $\theta_{\mathrm{ff}}$ is the free-free cooling coefficient (see Appendix). The last term of (36) represents expansion cooling. In the actual calculation we also include Compton heating by scattering of high energy photons off electrons, although it is not important since the reheating occurs at low redshifts $(z<100)$ and the energy of photons is small $(\lesssim \mathrm{keV})$ in our model. Compton cooling, among other cooling processes, is most important at $z \gtrsim 5$; the electron temperature is basically determined by the balance between Compton cooling and photoelectric ionization heating. At a lower redshift expansion cooling dominates.

\section{Results and implications}

The results of our calculation are presented in a few panels of Fig.3. Fig. 3(a) shows a fraction of HI and HII; the universe is reionized at $z \approx 30$, shortly after the first objects with mass of $\sim 10^{6} M_{\odot}$ collapse. By this epoch the relative abundance of

the baryons collapsed into stars is $\Omega_{\mathrm{star}} / \Omega_{B} \simeq 10^{-5}$ and the photon energy density is $\Omega_{\gamma} \simeq 10^{-10}$. Since then ionization is kept at a high degree; the HI fraction decreases below $10^{-6}$ by $z \simeq 20$ and stays at a value $<10^{-7}$ to the present epoch, which explains the very strong limit on neutral hydrogen from the Gunn-Peterson test (Gunn \& Peterson 1965; Steidel and Sargent 1989; Jenkins \& Ostriker 1991; Webb et al 1992). If we would switch off quasars, the hydrogen tends to recombine towards $z \approx 1-2$ $\left[n(\mathrm{HI}) / n_{\mathrm{H}} \approx 10^{-4}\right.$ at $\left.z=0\right]$. However, the uv flux from quasars, which appears as a kink in the curve of HII around $z \approx 6$ when they turn on, ensures continuous high ionization to $z=0$. We remark that the absorption due to possible Lyman $\alpha$ clouds at high $z$ might reduce the ionizing flux and lead to more HI (Miralda-Escudé \& Ostriker 1992; Madau 1993), which we ignore in the present work however.

Whether anisotropies with angular scale smaller than a few degrees may be lessened in our reheating scenario is an interesting issue of the model. The Thomson 
optical depth calculated as

$$
\tau_{\mathrm{T}}=\int_{0}^{\infty} d t c \sigma_{\mathrm{T}} n_{e}
$$

is $\tau_{\mathrm{T}} \simeq 0.22$ for the ionization history shown in Fig.3(a) (the effect of helium is also taken into account). While this optical depth is not so large, a calculation made by Sugiyama, Vittorio \& Silk (1993) indicates that the Doppler peak of the Fourier component of $\mathrm{CMB}$ correlation function $c_{\ell}$ at $\ell \simeq 200$ decreases by a factor 2 and also the power for $\sim 10^{\prime}$ by a factor of 3 for reionization at $z \approx 30$. Their calculations also show that anisotropies at 1 degree scale are appreciably smaller than $1 \times 10^{-5}$, and are perfectly allowed by the limit from the South Pole II experiment (Gaier et al. 1992).

The Compton $y_{\mathrm{c}}$ parameter is also plotted in the same figure (Fig.3(a)). The final value is $2 \times 10^{-7}$. This small $y_{\mathrm{c}}$ is understood by the fact that ionization takes place at low redshift with low energy photons, which keep electrons to stay at a low temperature; hence only a small fraction of energy goes into CMB by Compton cooling. This contrasts the model of Gnedin \& Ostriker (1991), where high energy photons are injected from accretion activity of massive black holes which are formed at a very high redshift; in their model $y_{\mathrm{c}}$ is as high as $10^{-4}$, though the predicted value is perhaps flexible. We should also add a remark on the difference of our model from the model with heating by shock waves; the latter heats up electrons to $1-10 \mathrm{keV}$ and leads to $y_{\mathrm{c}}$-parameter as large as $>10^{-3}$ (Yoshioka \& Ikeuchi 1987); shock heating is allowed only at small $z$ ( for this class of model, see Cen \& Ostriker 1992).

The metal abundance $Z$ (Fig.3(b)) closely follows the star abundance. For $z \lesssim 10$, $Z$ stays at $(0.4-1) \times 10^{-3}$, which is a typical metal abundance of population II stars. This means that our fractional star-formation parameter $f \simeq 2 \times 10^{-2}$ is close to the limit; namely, the observed metal abundance limits the amount of energy injection from stars.

Ionization history of helium atoms is exhibited in Fig.3(c). He I is ionized to He II $(\Delta E=24.6 \mathrm{eV})$ at the epoch of hydrogen reionization, and $n(\mathrm{HeII}) / n_{\mathrm{He}} \simeq 10^{-5}$ after 
this redshift. He II is then fully ionized to He III $(\Delta E=54.4 \mathrm{eV})$ when quasars turn on. Since the starlight can not ionize He II, the abundance of He III is taken as an indicator for the importance of the quasar light (Mo, Miralda-Escudé \& Rees 1993).

In Fig.3(d) we plot the electron temperature, the baryon Jeans mass (10), and the critical mass for the cooling condition (9). We note that the electron temperature stays between $3 \times 10^{3}-2 \times 10^{4} \mathrm{~K}$ for all period of $z<30$. The fact that the universe is fully ionized despite this low temperature can be understood by the slow recombination rate.

One of the most conspicuous effect in the reionized universe is a large Jeans mass at low redshift (Couchman \& Rees 1986). It gradually increases from $10^{4} M_{\odot}$ for $z<100$ to $1 \times 10^{9} M_{\odot}$ for $z<6$. This means that normal galaxies formed at $z<10$ should have a mass function with a peak around $M_{\text {luminous }} \approx 10^{9}-10^{10}$, or a luminosity function with a peak at a $B$ magnitude $M_{B} \approx-19$ mag rather than obey a Schechter function. Observationally, little is known about the faint end of the luminosity function of field galaxies. The only information available on the faint end is from galaxies in the Virgo cluster; Binggeli, Sandage \& Tammann (1985) obtained a complete galaxy sample down to $B=18 \mathrm{mag}$, and they have shown that the luminosity function of spiral and elliptical galaxies are of the Gaussian type with peaks at $M_{B}=-18.1 \mathrm{mag}$ (dispersion $\sigma=1.5 \mathrm{mag}$ ) and $M_{B}=-18.2 \mathrm{mag}(\sigma=1.7 \mathrm{mag})$, respectively (Sandage, Binggeli \& Tammann 1985). [Here we used $\left\langle(m-M)_{0}\right\rangle \simeq 31.4$ for spiral galaxies and 31.0 for elliptical galaxies; see the discussion in Fukugita, Okamura \& Yasuda (1993).] The Jeans mass curve shown in Fig.3(d) suggests that very small galaxies, such as dwarf spheroidals, and globular clusters are very early objects which collapsed at $z>10$ and have survived merging to larger systems.

The critical cooling mass is smaller than the Jeans mass in the reheated universe, in contrast to the case without reionization, for which generally $M_{B, c}>M_{B, J}$.

The resulting photon spectrum is given in Fig. 4 for $z=5,3$ and 0 . The relative contributions from stars and quasars are obvious in this figure. We show in Fig. 5 
the strength of ionizing flux defined by

$$
\bar{J}=\int_{\epsilon_{\mathrm{HI}}}^{\infty} \frac{d \epsilon_{\gamma}}{4 \pi c} n_{\gamma}\left(\epsilon_{\gamma}\right) \frac{\sigma_{1 \mathrm{f}, \mathrm{HI}}\left(\epsilon_{\gamma}\right)}{\sigma_{1 \mathrm{f}, \mathrm{HI}}\left(\epsilon_{\mathrm{HI}}\right)}
$$

as a function of redshift. The ionizing flux rises very sharply at $z \simeq 30$, the epoch of reionization. As we noted earlier the flux at low redshift may have substantial uncertainties, arising from our underestimate of stationally star formation activity in normal galaxies and from the neglect of the absorption by Lyman $\alpha$ clouds. Nevertheless, the value of $\bar{J}$ around $z=5-3\left(\bar{J}=(3-5) \times 10^{-21} \mathrm{erg} \mathrm{cm}^{-2} \mathrm{~s}^{-1} \mathrm{sr}^{-1} \mathrm{~Hz}^{-1}\right)$ is interesting, since it is about the value required to keep Lyman $\alpha$ clouds from their collapse (Sargent et al 1980; Ostriker \& Ikeuchi 1983; Rees 1986; Bajtlik, Duncan \& Ostriker 1988).

\section{Conclusion and discussion}

We have shown that OB stars which would have been formed shortly after the collapse of very early objects, as expected in the standard CDM model, ionize the universe completely at the epoch as early as $z \approx 30$. This reionization significantly lessens the anisotropies of the CMB at an angular scale smaller than $1^{\circ}$ and removes the marginal conflict between the COBE-DMR data and the limit from the South Pole II experiment in the CDM model (e.g., Górski, Stompor \& Juszkiewicz 1992), without invoking the aid of the tensor perturbations (Crittenden, R. et al. 1993; Davis, R.L. et al. 1992; Dolgov \& Silk 1992). Reionization has also an interesting implication, that luminosity function of normal galaxies formed at $z<10$ should have a cutoff on the faint side, which roughly corresponds to the luminosity of dwarf galaxies. Smaller

bound systems such as globular clusters and dwarf spheroidals must be the objects formed very early in the universe.

Another advantage of the present scenario is that the metal abundance of globular clusters can be explained naturally. The observed metal abundance sets an upper limit on the photon energy that is emitted from stars and injected into the universe. 
We have shown that the null result of the Gunn-Peterson(GP) test is satisfied by reheating in contrast to the case of shock wave reheating as advocated by Cen and Ostriker 1992; with shock heating alone ionization is not so efficient and the GP test is not satisfied by many orders of magnitude. The GP test is not satisfied in a heating model with very early formation of massive black holes (Gnedin \& Ostriker 1992); the light emitted from an early epoch is redshifted away and has no ionizing power at low redshift. In order to keep hydrogen highly ionized, the ionizing uv flux must exist during the relevant epochs. This is satisfied in our model by successive formation of bound objects. We also expect the ionizing flux as strong as is required to confine Lyman $\alpha$ clouds by continuous ionization.

In our reheating scenario the Compton $y_{\mathrm{c}}$ parameter takes a very small value, much smaller than could be detected by the COBE-FIRAS experiment (Mather et al. 1993).

The most interesting test for the present case is the CMB anisotropies at a small angular scale. If anisotropies would be observed, for instance, at a level of $\Delta T / T \approx$ $1 \times 10^{-5}$ reheating must not have happened before $z \approx 10-15$, which means that the baryon fraction that goes into stars should be smaller than $f \simeq 10^{-3}$ in our terminology.

All of our calculation, except for the normalization of the fluctuations, scales as $\Omega h^{2}$ (and $\Omega_{B} h^{2}$ ), and the normalization of the fluctuations depends very weakly on $\Omega\left(\Omega^{-0.2}\right)$. Therefore, the reheating scenario changes little if high value of the Hubble constant is taken in a low density universe. If $\Omega h^{2} \ll 1$, however, the epoch of reheating is considerably delayed.

Our calculation presented here is certainly far from complete for thermal history and evolution of galaxies after they are formed. We have to carry out a calculation with a more astronomical treatment to take proper account of the effects.

\section{Acknowledgement}


We would like to thank Martin Rees for his valuable comments. 


\section{A Quantitative argument for heating by collapsing bound objects}

When the matter collapses, baryon gas and dark matter particles are virialized and distributed isothermally with radius $r_{\text {vir }}$ and temperature $T_{\text {vir }}$, which are given by

$$
\begin{aligned}
r_{\mathrm{vir}} & =\left[\frac{M}{6 \pi^{3} \rho_{\mathrm{vir}}}\right]^{1 / 3} \\
& =1.7 \times 10^{-5} \mathrm{Mpc}\left(\frac{M}{M_{\odot}}\right)^{1 / 3}(1+z)^{-1}\left(\Omega h^{2}\right)^{-1 / 3}
\end{aligned}
$$

and

$$
\begin{aligned}
T_{\mathrm{vir}} & =\frac{G m_{p} M \mu}{3 r_{\mathrm{vir}}} \\
& =1.04 \times 10^{-2} \mathrm{~K} \mu\left(\frac{M}{M_{\odot}}\right)^{2 / 3}(1+z)\left(\Omega h^{2}\right)^{1 / 3},
\end{aligned}
$$

where $\rho_{\text {vir }}$ is the mean mass density of the virialized object and we take $\rho_{\text {vir }}=180 \rho$ from the spherical collapse model. The temperature of the baryon gas decreases by

radiative cooling processes, which convert the kinetic energy of baryon gas into radiation. If free-free cooling or recombination cooling dominates over other cooling processes, the produced photons could have energy high enough to ionize the intergalactic medium.

This scenario requires that free-free or recombination cooling must be more efficient than Compton cooling, expansion cooling and line cooling. For fully ionized plasma, the cooling times for the free-free and recombination processes are

$$
\begin{aligned}
\tau_{\mathrm{ff}} & =\frac{3 T_{e} n_{B}}{2 \mu \Lambda_{\mathrm{ff}}} \\
& \simeq 1.66 \times 10^{14} \mathrm{sec}(T / \mathrm{K})^{1 / 2}(1+z)^{-3}\left(\Omega_{B} h^{2}\right)^{-1}
\end{aligned}
$$

and

$$
\tau_{\text {rec }}=\frac{3 T_{e} n_{B}}{2 \mu \Lambda_{\text {rec }}}
$$




$$
\simeq 1.19 \times 10^{13} \sec \left(\frac{T}{\mathrm{~K}}\right)^{0.7}\left[1+\left(\frac{T}{10^{6} \mathrm{~K}}\right)^{0.7}\right](1+z)^{-3}\left(\Omega_{B} h^{2}\right)^{-1},
$$

where the cooling rates $\Lambda_{\mathrm{ff}}$ and $\Lambda_{\mathrm{rec}}$ are given by

$$
\begin{aligned}
\Lambda_{\mathrm{ff}}= & 1.42 \times 10^{-27} g_{\mathrm{ff}}\left(T_{e} / \mathrm{K}\right)^{1 / 2}(n(\mathrm{HII})+4 n(\mathrm{HeIII})) n_{e} \mathrm{erg} \mathrm{cm}^{-3} \mathrm{sec}^{-1}, \\
\Lambda_{\mathrm{rec}}= & 6.5 \times 10^{-27}\left(\frac{T_{e}}{\mathrm{~K}}\right)^{1 / 2}\left(\frac{T_{e}}{10^{3} \mathrm{~K}}\right)^{-0.2}\left[1+\left(\frac{T_{e}}{10^{6} \mathrm{~K}}\right)^{0.7}\right]^{-1} \\
& \times n(\mathrm{HII}) n_{e} \mathrm{erg} \mathrm{cm}^{-3} \mathrm{sec}^{-1}
\end{aligned}
$$

with $n_{e}, n$ (HII) and $n$ (HeIII) the number densities of electron, HII and HeIII, and $g_{\text {ff }}$ the gaunt factor of $\sim O(1)$. That the cooling time $\tau_{\text {ff }}$ or $\tau_{\text {rec }}$ be shorter than the Compton cooling time $\tau_{\mathrm{C}}=3.9 \times 10^{19}(1+z)^{-4}$ sec leads to the conditions:

$$
\begin{aligned}
\frac{\tau_{\mathrm{C}}}{\tau_{\text {ff }}} & =29\left(\frac{T_{e}}{10^{4} \mathrm{~K}}\right)^{-1 / 2}(1+z)^{-1}\left(\frac{\Omega_{B} h^{2}}{0.0125}\right)>1, \\
\frac{\tau_{\mathrm{C}}}{\tau_{\text {rec }}} & =65\left(\frac{T_{e}}{10^{4} \mathrm{~K}}\right)^{-0.7}\left[1+\left(\frac{T_{e}}{10^{6} \mathrm{~K}}\right)^{0.7}\right]^{-1}(1+z)^{-1}\left(\frac{\Omega_{B} h^{2}}{0.0125}\right)>1 .
\end{aligned}
$$

The cooling times $\tau_{\text {ff }}$ or $\tau_{\text {rec }}$ should also be shorter than the dynamical time $\tau_{H}=$ $1 / H=(\dot{a} / a)^{-1}$ to make the cooling processes effective.

$$
\begin{aligned}
\frac{H^{-1}}{\tau_{\text {ff }}}= & 0.45\left(\frac{T_{e}}{10^{4} \mathrm{~K}}\right)^{-1 / 2}(1+z)^{3 / 2}\left(\frac{\Omega_{B} h^{2}}{0.0125}\right)\left(\frac{\Omega h^{2}}{0.25}\right)^{-1 / 2}>1, \\
\frac{H^{-1}}{\tau_{\text {rec }}}= & 0.99\left(\frac{T_{e}}{10^{4} \mathrm{~K}}\right)^{-0.7}\left[1+\left(\frac{T_{e}}{10^{6} \mathrm{~K}}\right)^{0.7}\right]^{-1}(1+z)^{3 / 2} \\
& \times\left(\frac{\Omega_{B} h^{2}}{0.0125}\right)\left(\frac{\Omega h^{2}}{0.25}\right)^{-1 / 2}>1 .
\end{aligned}
$$

These condition (45)-(48) are satisfied simultaneously for temperature $T<3 \times 10^{5} \mathrm{~K}$, as seen in Fig.2, where the critical temperature for $\tau_{\mathrm{C}}=\tau_{\text {ff }}$ etc. are plotted as a function of redshift.

Another condition to produce efficiently ionizing uv photons is that the recombination cooling rate must dominate over the rate of line cooling of hydrogen atoms 
that are excited collisionally. The latter rate is given by

$$
\Lambda_{\text {line }}=7.5 \times 10^{-19}\left[1+\left(\frac{T_{e}}{10^{5} \mathrm{~K}}\right)^{1 / 2}\right]^{-1} \exp \left(-\frac{1.18 \times 10^{5}}{T_{e}}\right) n_{e} n(\mathrm{HI}) \mathrm{erg} \mathrm{cm}^{-3} \mathrm{sec}^{-1}
$$

so that the ratio $\Lambda_{\text {line }} / \Lambda_{\text {rec }}$ is

$$
\begin{aligned}
\frac{\Lambda_{\text {line }}}{\Lambda_{\text {rec }}} & =2.9 \times 10^{7}\left(\frac{T_{e}}{\mathrm{~K}}\right)^{-0.3}\left(1+\left(\frac{T_{e}}{10^{5} \mathrm{~K}}\right)^{1 / 2}\right)^{-1} \\
& \times\left(1+\left(\frac{T_{e}}{10^{6} \mathrm{~K}}\right)^{0.7}\right) \frac{n(\mathrm{HI})}{n(\mathrm{HII})} \exp \left(\frac{-1.18 \times 10^{5} \mathrm{~K}}{T_{e}}\right) .
\end{aligned}
$$

The condition $\Lambda_{\text {line }} / \Lambda_{\text {rec }}<1$ means that the fraction of HI be small enough. Fig.6 shows the contour of $\Lambda_{\text {line }} / \Lambda_{\text {rec }}=1$ in the plane of $T$ versus $[n(\mathrm{HI}) / n(\mathrm{HII})]$; the production of photons requires that the parameters should lie below this contour. On the other hand, $n(\mathrm{HI}) / n(\mathrm{HII})$ is determined by ionization equilibrium, as

$$
\begin{aligned}
\frac{n(\mathrm{HI})}{n(\mathrm{HII})} & =4.95\left(\frac{T_{e}}{\mathrm{~K}}\right)^{-1.2}\left[1+\left(\frac{T_{e}}{10^{5} \mathrm{~K}}\right)^{0.5}\right] \\
& \times\left[1+\left(\frac{T_{e}}{10^{6} \mathrm{~K}}\right)^{0.7}\right]^{-1} \exp \left(\frac{1.58 \times 10^{5} \mathrm{~K}}{T_{e}}\right) .
\end{aligned}
$$

The curve showing the equilibrium condition is also plotted in Fig.6. One can see that the temperature should be greater than $3 \times 10^{5} \mathrm{~K}$ for recombination cooling to dominate over line cooling. However, this is just opposite to the condition for recombination cooling being faster than Compton cooling. This proves that liberation of energy in bound objects does not efficiently produce ionizing photons.

\section{B Ionization and recombination coefficients}

The ionization and recombination coefficients used in our calculation is summarized in this Appendix. The coefficients and cross sections used in our paper are taken from Black 1981; Matsuda et al. 1971; Menzel \& Pekeris 1935; Spitzer 1978. We adopt the high temperature correction given by Cen 1992 for the coefficients of processes involving a free electron and an orbital electron. 


\section{B.1 Collisional ionization}

(a) HI $(n=1 \rightarrow$ free):

$$
\beta_{1, \mathrm{HI}}=5.85 \times 10^{-11} T^{1 / 2}\left(1+\left(T / 10^{5}\right)^{0.5}\right)^{-1} \exp \left(-1.578 \times 10^{5} / T\right) \mathrm{cm}^{3} \mathrm{sec}^{-1},
$$

(b) HI ( $n=2 \rightarrow$ free):

$$
\begin{aligned}
\beta_{2, \mathrm{HI}} & =5.46 \times 10^{-11} T^{1 / 2} \exp \left(-3.882 \times 10^{4} / T\right) \\
& \times\left[19.98-5.89 \times 10^{-5} \mathrm{~T}-2.81 \times 10^{4} T^{-1}+5.44 \times 10^{7} T^{-2}\right] \mathrm{cm}^{3} \mathrm{sec}^{-1},(53)
\end{aligned}
$$

(c) HeI:

$$
\beta_{\mathrm{HeI}}=2.38 \times 10^{-11} T^{1 / 2}\left(1+\left(T / 10^{5}\right)^{0.5}\right)^{-1} \exp \left(-2.853 \times 10^{5} / T\right) \mathrm{cm}^{3} \mathrm{sec}^{-1},
$$

(d) HeII:

$$
\beta_{\mathrm{HeII}}=5.68 \times 10^{-12} T^{1 / 2}\left(1+\left(T / 10^{5}\right)^{0.5}\right)^{-1} \exp \left(-6.315 \times 10^{5} / T\right) \mathrm{cm}^{3} \mathrm{sec}^{-1} .
$$

\section{B.2 Recombination}

(a) HII (free $\rightarrow n \geq 1)$ :

$$
\alpha_{\mathrm{HII}}=6.28 \times 10^{-11} T^{-1 / 2}\left(\frac{T}{10^{3}}\right)^{-0.2}\left[1+\left(\frac{T}{10^{5}}\right)^{0.7}\right]^{-1} \mathrm{~cm}^{3} \mathrm{sec}^{-1},
$$

(b) HII (free $\rightarrow n \geq 2$ ) [Peebles 1993]:

$$
\alpha_{2, \mathrm{HII}}=2.6 \times 10^{-13}\left(\frac{T}{10^{4}}\right)^{-0.8} \mathrm{~cm}^{3} \mathrm{sec}^{-1},
$$

(c) HeII (free $\rightarrow n \geq 1$ ):

$$
\alpha_{\mathrm{HeII}}=1.50 \times 10^{-10} T^{-0.6353} \mathrm{~cm}^{3} \mathrm{sec}^{-1},
$$

(d) HeII (free $\rightarrow n^{3}[n \geq 2]$ )

$$
\alpha_{\mathrm{HeII}, 2^{3}}=9.94 \times 10^{-11} T^{-0.6687} \mathrm{~cm}^{3} \mathrm{sec}^{-1},
$$


(e) HeII (free $\rightarrow 1^{1} S$ )

$$
\alpha_{\mathrm{HeII}, 1^{1} S}=1.32 \times 10^{-11} T^{-0.480} \mathrm{~cm}^{3} \mathrm{sec}^{-1},
$$

(f) HeII (dielectronic recombination):

$$
\begin{aligned}
\xi_{\text {HeII }}= & 1.9 \times 10^{-3} T^{-1.5} \exp \left(-4.7 \times 10^{5} / T\right) \\
& \times\left[1+0.3 \exp \left(-9.4 \times 10^{4} / T\right)\right] \mathrm{cm}^{3} \mathrm{sec}^{-1},
\end{aligned}
$$

(g) HeIII (free $\rightarrow n \geq 1$ ):

$$
\alpha_{\text {HeIII }}=3.36 \times 10^{-10} T^{-1 / 2}\left(\frac{T}{10^{3}}\right)^{-0.2}\left[1+\left(\frac{T}{4 \times 10^{6}}\right)^{0.7}\right]^{-1} \mathrm{~cm}^{3} \sec ^{-1},
$$

(h) HeIII (free $\rightarrow m \geq 2$ ):

$$
\alpha_{\mathrm{HeIII}, 2}=\left\{\begin{array}{ll}
\alpha_{\mathrm{HeIII}}(1.11-0.044 \ln T) & T<2.2 \times 10^{4} \\
\alpha_{\mathrm{HeIII}}(1.43-0.076 \ln T) & T>2.2 \times 10^{4}
\end{array},\right.
$$

\section{B.3 Photoionization cross sections}

(a) HI $(n=1 \rightarrow$ free $)$ :

$$
\sigma_{\mathrm{bf}, \mathrm{HI}}=1.18 \times 10^{-11} \epsilon_{\gamma}^{-4} \frac{e^{-4\left(\arctan z_{1}\right) / z_{1}}}{1-e^{-2 \pi / z_{1}}} \mathrm{~cm}^{2}
$$

where $z_{1}=\left[\epsilon_{\gamma} / \epsilon_{\mathrm{HI}}-1\right]^{1 / 2}$.

(b) HI $(n=2 \rightarrow$ free):

$$
\sigma_{\mathrm{bf}, \mathrm{HI}, 2}=1.08 \times 10^{-13} \epsilon_{\gamma}^{-3} \frac{\left(3+4 z^{2}\right)\left(5+4 z^{2}\right) e^{-4(\arctan (2 z)) / z}}{\left(1+4 z^{2}\right)^{3}\left(1-e^{-2 \pi / z}\right)} \mathrm{cm}^{2},
$$

where $z=\left[\epsilon_{\gamma} / \epsilon_{\mathrm{HI}}-1 / 4\right]^{2}$.

(c) HeI:

$$
\sigma_{\mathrm{bf}, \mathrm{HeI}}=1.13 \times 10^{-14}\left(\frac{1}{\epsilon_{\gamma}^{2.05}}-\frac{9.775}{\epsilon_{\gamma}^{3.05}}\right) \mathrm{cm}^{2},
$$

(d) HeII:

$$
\sigma_{b f, \mathrm{HeII}}=7.55 \times 10^{-10} \epsilon_{\gamma}^{-4} \frac{e^{-4\left(\arctan z_{2}\right) / z_{2}}}{1-e^{-2 \pi / z_{2}}} \mathrm{~cm}^{2}
$$

where $z_{2}=\left[\epsilon_{\gamma} / \epsilon_{\mathrm{HeII}}-1\right]^{1 / 2}$. 


\section{B.4 Cooling Rates}

\section{B.4.1 Collisional ionization cooling}

(a) HI:

$$
\zeta_{\mathrm{HI}}=1.27 \times 10^{-21} T^{1 / 2}\left[1+\left(\frac{T}{10^{5}}\right)^{1 / 2}\right]^{-1} \exp \left(-1.58 \times 10^{5} / T\right) \mathrm{erg} \mathrm{cm}^{3} \mathrm{sec}^{-1},
$$

(b) HeI:

$$
\zeta_{\text {HeI }}=9.38 \times 10^{-22} T^{1 / 2}\left[1+\left(\frac{T}{10^{5}}\right)^{1 / 2}\right]^{-1} \exp \left(-2.85 \times 10^{5} / T\right) \mathrm{erg} \mathrm{cm}^{3} \mathrm{sec}^{-1},
$$

(c) $\operatorname{HeI}\left(2^{3} S\right)$ :

$$
\begin{aligned}
\zeta_{\mathrm{HeI}\left(2^{3} S\right)} n(\mathrm{HeI}) & =5.01 \times 10^{-27} T^{-0.1687}\left[1+\left(\frac{T}{10^{5}}\right)^{1 / 2}\right]^{-1} \\
& \times \exp \left(-5.53 \times 10^{4} / T\right) n_{e} n(\mathrm{HeII}) \mathrm{erg} \mathrm{sec}^{-1} .
\end{aligned}
$$

(d) HeII:

$$
\zeta_{\text {HeII }}=4.95 \times 10^{-22} T^{1 / 2}\left[1+\left(\frac{T}{10^{5}}\right)^{1 / 2}\right]^{-1} \exp \left(-6.31 \times 10^{5} / T\right) \mathrm{erg} \mathrm{cm}^{3} \mathrm{sec}^{-1},
$$

\section{B.4.2 Recombination cooling}

(a) HII:

$$
\eta_{\mathrm{HII}}=6.50 \times 10^{-27} T^{1 / 2}\left(\frac{T}{10^{3}}\right)^{-0.2}\left[1+\left(\frac{T}{10^{6}}\right)^{0.7}\right]^{-1} \mathrm{erg} \mathrm{cm}^{3} \mathrm{sec}^{-1},
$$

(b) HeII:

$$
\eta_{\text {HeII }}=1.55 \times 10^{-26} T^{0.3647} \mathrm{erg} \mathrm{cm}^{-3} \mathrm{sec}^{-1},
$$

(c) HeII (dielectronic recombination)

$$
\begin{aligned}
\omega_{\text {HeII }}= & 1.24 \times 10^{-13} T^{-1.5} \exp \left(-4.7 \times 10^{5} / T\right) \\
& \times\left[1+0.3 \exp \left(-9.4 \times 10^{4} / T\right)\right] \mathrm{erg} \mathrm{cm}^{3} \mathrm{sec}^{-1},
\end{aligned}
$$

(d) HeIII:

$$
\eta_{\text {HeIII }}=3.48 \times 10^{-26} T^{1 / 2}\left(\frac{T}{10^{3}}\right)^{-0.2}\left[1+\left(\frac{T}{4 \times 10^{6}}\right)^{0.7}\right]^{-1} \mathrm{erg} \mathrm{cm}^{3} \mathrm{sec}^{-1} .
$$




\section{B.4.3 Collisional excitation cooling}

(a) HI:

$$
\psi_{\mathrm{HI}}=7.5 \times 10^{-19}\left[1+\left(\frac{T}{10^{5}}\right)^{1 / 2}\right]^{-1} \exp \left(-1.18 \times 10^{5} / T\right) \mathrm{erg} \mathrm{cm}^{3} \mathrm{sec}^{-1},
$$

(b) $\mathrm{HeI}$

$$
\begin{aligned}
\psi_{\mathrm{HeI}} n(\mathrm{HeI}) & =9.10 \times 10^{-27} T^{-0.1687}\left[1+\left(\frac{T}{10^{5}}\right)^{1 / 2}\right]^{-1} \\
& \times \exp \left(-1.31 \times 10^{4} / T\right) n_{e} n(\mathrm{HeII}) \mathrm{erg} \sec ^{-1},
\end{aligned}
$$

(c) HeII:

$$
\psi_{\mathrm{HeII}}=5.54 \times 10^{-17} T^{-0.397}\left[1+\left(\frac{T}{10^{5}}\right)^{1 / 2}\right]^{-1} \exp \left(-4.73 \times 10^{5} / T\right) \mathrm{erg} \mathrm{cm}^{3} \mathrm{sec}^{-1}
$$

\section{B.4.4 Free-free cooling}

$$
\theta_{\mathrm{ff}}=1.42 \times{ }^{-27} g_{\mathrm{ff}} T^{1 / 2}
$$

\section{B.4.5 Compton cooling}

$$
\lambda_{\mathrm{c}}=4 k\left(T_{e}-T_{\gamma}\right) \frac{\pi^{2}}{15}\left(\frac{k T}{\hbar c}\right)^{3}\left(\frac{k T}{m_{e} c^{2}}\right) n_{e} \sigma_{\mathrm{T}} c
$$




\section{References}

Adams, F., Bond, J.R, Freeses, K. Freeman, J., \& Olinto, A. 1992, Phys. Rev. D47, 426.

Bajtlik, S., Duncan, R.C. \& Ostriker, J.P., 1988, ApJ 327, 570.

Barcons, X., Fabian, A.C. \& Rees, M.J. 1991, Nat 350, 685.

Bardeen, J.M., Bond, J.R., Kaiser, N. \& Szalay, A.S. 1986, ApJ 304, 15.

Bartlett, J.G. \& Stebbins, A. 1991, ApJ 371, 8.

Binggeli, B., Sandage, A. \& Tammann, G. A. 1985, AJ 90, 1681.

Black, J.H. 1981, MNRAS 197, 553.

Blumenthal, G.R., Faber, S.M., Primack, J.R. \& Rees, M.J. 1984, Nat 311, 517.

Bond, J.R., Carr, B.J. \& Hogan, C.J. 1986, ApJ 306, 428.

Bond, J.R. \& Szalay, A.S. 1983, ApJ 274, 443.

Carr, B.J., Bond, J.R. \& Arnett, W.D. 1984, ApJ 277, 445.

Cen, R. 1992, ApJS 78, 341.

Cen, R., Jameson, A., Liu, A. \& Ostriker, J.P. 1990, ApJ 362, L41.

Cen, R. \& Ostriker, J.P. 1992, ApJ 399, 331.

Couchman, H.M.P. 1985, MNRAS 214, 137.

Couchman, H.M.P. \& Rees, M.J. 1986, MNRAS 214, 137.

Crittenden, R., Bond, J.R., Davis, R.L., Efstatiou, G. \& Steinhardt, P.J. 1993, Phys. Rev. Lett. $71,324$. 
Davis, M, Summers, F. \& Schlegel, D. 1992, Nat 359, 393.

Davis, R.L., Hodges, H.M., Smoot, G.F., Steinhardt \& Turner, M.S. 1992, Phys. Rev. Lett. 69, 1856.

Doglov, A.D. \& Silk, J. 1992, Berkeley preprint.

Doroshkevich, Zeldovich, Ya. B. \& Novikov, I. D. 1967, Astr. Zh. 44, 295 [Soviet Astron. - AJ 11, 233].

Efstathiou, G., Bond, J. R. \& White, S. D. M. 1992, MNRAS 258, 1p.

Efstathiou, G. \& Rees, M.J. 1988, MNRAS 230, 5p.

Fukugita, M. \& Kawasaki, M. 1990, ApJ 353, 384.

Fukugita, M. \& Kawasaki, M. 1993, ApJ 402, 58.

Fukugita, M., Okamura, S. \& Yasuda, N. 1993, ApJ 412, L13

Frenk, C.S., White, S.D.M., Davis, M. \& Efstathiou, G. 1988, ApJ 327, 507.

Gaier, T., et al. 1992, ApJ 398, L1.

Gnedin, N.Y. \& Ostriker, J.P. 1992, ApJ 400, 1.

Górski, K., Stompor, R. \& Juszkiewicz, R. 1992, YITP preprint.

Gunn, J.E. \& Peterson, B.A. 1965, ApJ 142, 1633.

Hogan, C.J. 1980, MNRAS 192, 891.

Jenkins, E.B. \& Ostriker, J.P. 1991, ApJ 376, 33.

Jones, B.J.T. \& Wyse, R.F.G. 1985, A\&A 149, 144.

Klypin, A., Holtzman, J., Primack, J. \& Rogos, E. 1992, ApJ in press. 
Madau, P. 1992, ApJ 389, L1.

Mather, J.C., et al. 1993, COBE preprint.

Matsuda, T., Sato, H., and Takeda, H. 1971, Prog. Theor. Phys. 46, 416.

Menzel, D.H. \& Pekeris, C.L. 1935, MNRAS 96, 77.

Miralda-Escudé, J. \& Ostriker, J.P. 1990, ApJ 350, 1.

Miralda-Escudé, J. \& Ostriker, J.P. 1992, ApJ 392, 15.

Mo, H. J., Miralda-escudé, J. \& Rees, M. 1993, Cambridge IoA preprint.

Ostriker, J.P. \& Ikeuchi, S. 1983, ApJ 263, L63.

Peebles, P.J.E. 1968, ApJ 153, 1.

Peebles, P.J.E. 1982, ApJ 263, L1.

Peebles, P.J.E. 1993, Principles of Physical Cosmology (Princeton University Press).

Press, W.H. \& Schechter, P.L. 1974, ApJ 181,425.

Rees, M.J. 1986, MNRAS 218, 25p.

Sandage, A., Binggeli, B. \& Tammann, G. A. 1985, ApJ 90, 1759

Sargent, W.L.W., Young, P.J., Boksenberg \& Tytler, D. 1980, ApJS 69, 703.

Sasaki, S., Takahara, F. \& Suto, Y. 1993, preprint YITP/U-93-02.

Sugiyama, N., Silk, J. \& Vittorio, N. 1993, ApJ in press.

Shapiro, S.L. \& Teukolsky, S.A. 1983, 'Black Holes, White Dwarfs, and Neutron Stars', (John Wiley and Sons) p9.

Smoot, G.F., et al. 1992, ApJ 396, L1. 
Spitzer, L., Jr. 1978, Physical Processes in the Interstellar Medium (New York: Wiley).

Stebbins, A. \& Silk, J. 1986, ApJ 300, 1.

Steidel, C.C. \& Sargent, W.L. 1989, ApJ 343, L33.

Yoshioka, S. \& Ikeuchi, S. 1987, ApJ 323, L7.

Walker, T.P., Steigman, G., Schramm, D.N., Olive, K.A. and Kang, H.-S. 1991, ApJ 376, 51 .

Webb, J.K., Barcons, X., Carswell, R.F. \& Parnell, H.C. 1992, MNRAS 255, 319.

White, S.D. \& Frenk, C.S. 1991, ApJ 379, 52. 


\section{Figure Captions}

Fig.1 (a) Evolution of the density of collapsed objects with mass $M$ greater than prescribed values (shown in units of $M_{\odot}$ ) for $\Omega=1, h=0.5$.

(b) Evolution of the mass fraction of stars for $\Omega_{B}=0.05$ and $f=0.02(\Omega$ and $h$ are the same as in (a)) with the effect of reionization taken into account (solid curve). The dashed curve represents the mass fraction of stars when the effect of reionization is not taken into account.

(c) Evolution of the radiation energy emitted from stars (dashed curve) and from quasars (dashed-dotted curve). The solid curve represents the sum.

(d) Comoving number density of quasars plotted against redshift for $t_{\mathrm{Q}}=$ $10^{8}$ year and $\epsilon_{\mathrm{Q}} F_{\mathrm{Q}}=10^{-5}$.

Fig.2 Critical temperatures for $\tau_{\text {rec }}=\tau_{\mathrm{C}}, \tau_{\mathrm{ff}}=\tau_{\mathrm{C}}, \tau_{\text {rec }}=H^{-1}, \tau_{\mathrm{ff}}=H^{-1}, \tau_{\mathrm{C}}=H^{-1}$ plotted as a function of redshift. Region for $\tau_{\text {rec }} \leq \tau_{\mathrm{C}}, H^{-1}$ or $\tau_{\mathrm{ff}} \leq \tau_{\mathrm{C}}, H^{-1}$ is needed for high energy photon emission from the collapsing objects. Another requirement that $\tau_{\text {rec }} \leq \tau_{\text {line }}$, which is estimated from Fig.6 below, is also shown. There are no regions that satisfy all the requirement.

Fig.3 (a) Evolution of the fraction of HI (solid curve), HII (dashed curve) and $y_{\mathrm{c}^{-}}$ parameter (dashed-dotted curve) shown for $\Omega=1, \Omega_{B}=0.05, h=0.5$ and $f=0.02$.

(b) Evolution of metalicity $Z$ as a function of redshift.

(c) Evolution of the fraction of HeI (solid curve), HeII(dashed curve) and HeIII (dashed-dotted curve).

(d) Evolution of electron temperature $T_{e}(\mathrm{~K})$, baryonic Jeans mass $M_{B, J}$, and the critical mass $M_{B, c}$ above which the molecular cooling time is shorter than the dyanmical time. $M_{B, J}$ and $M_{B, c}$ are in units of $M_{\odot}$.

Fig.4 Residual photon spectra at $z=5$ (solid curve), $z=3$ (dashed curve) and $z=0$ (dashed-dotted curve) for $\Omega=1, \Omega_{B}=0.05, h=0.5$ and $f=0.02$. 
The dotted curve that closely follows the curve for $z=5$ stands for the spectrum $(z=5)$ when the effect of absorption due to intergalactic $\mathrm{H}$ and $\mathrm{He}$ would be switched off.

Fig.5 Evolution of the ionizing flux from stars and quasars shown for $\Omega=1, \Omega_{B}=$ 0.05, $h=0.5$ and $f=0.02$ (solid curves). Dashed curves represent the ionizing fluxes which are obtained when the effect of absorption due to $\mathrm{H}$ and He is switched off.

Fig.6 Condition for recombination cooling to dominate over line cooling for $\Omega=1$, $\Omega_{B}=0.05$ and $h=0.5$. The region below the solid line shows the parameters for which recombination cooling dominates. The dashed curve shows the ratio $n(\mathrm{HI}) / n(\mathrm{HII})$ from ionization equilibrium. 


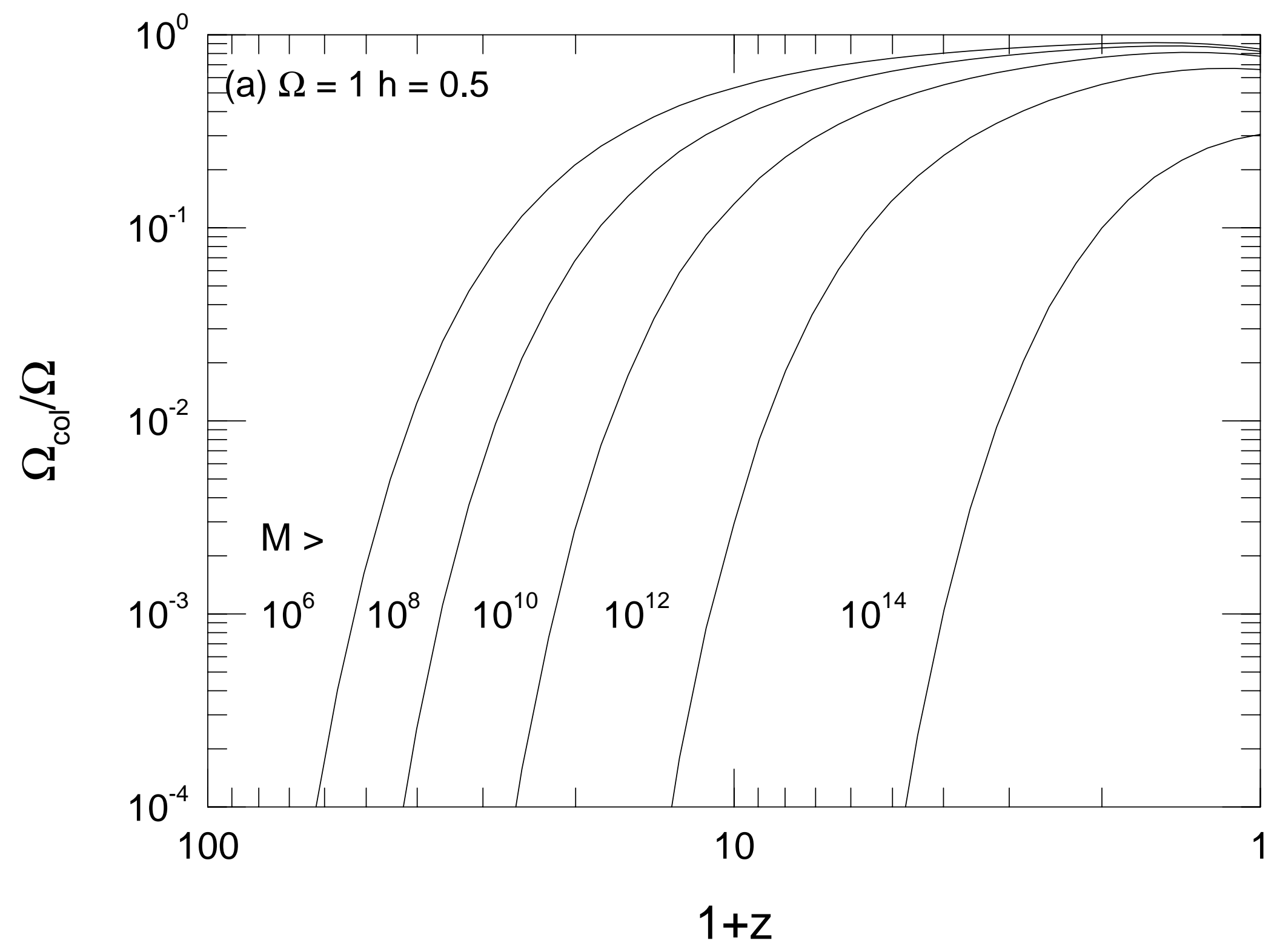

Fig.1(a) 


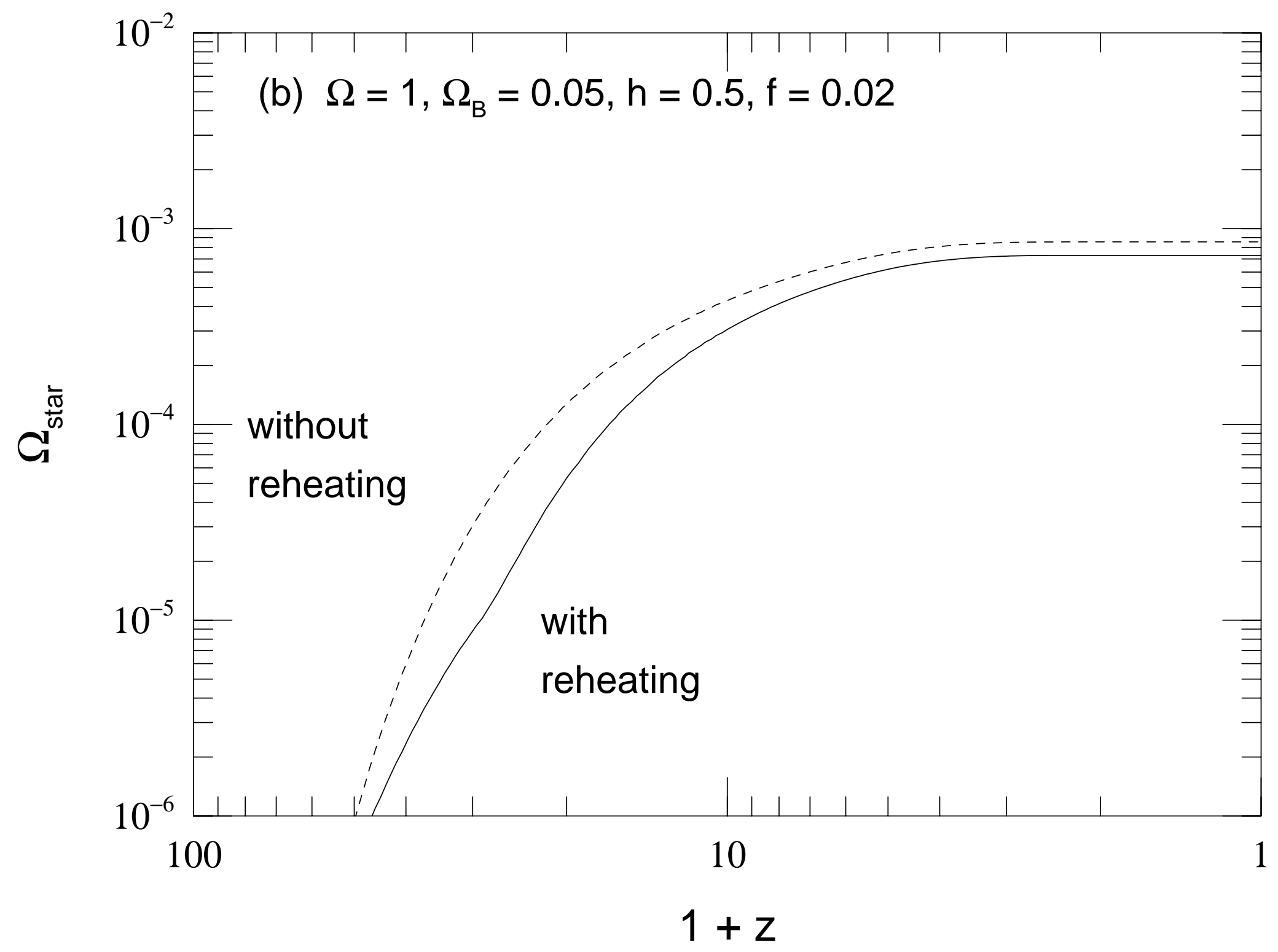

Fig.1(b) 


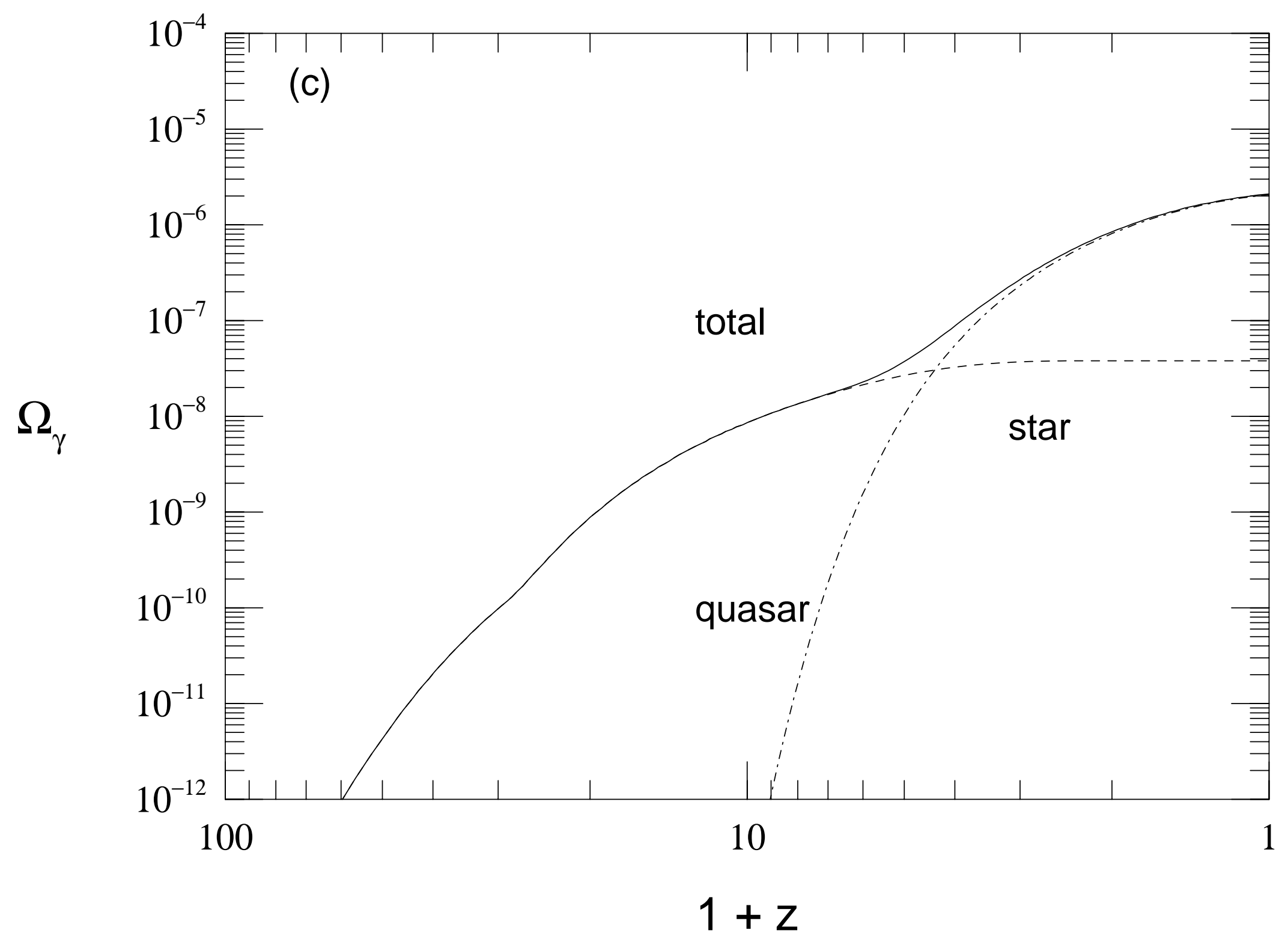

Fig.1(c) 


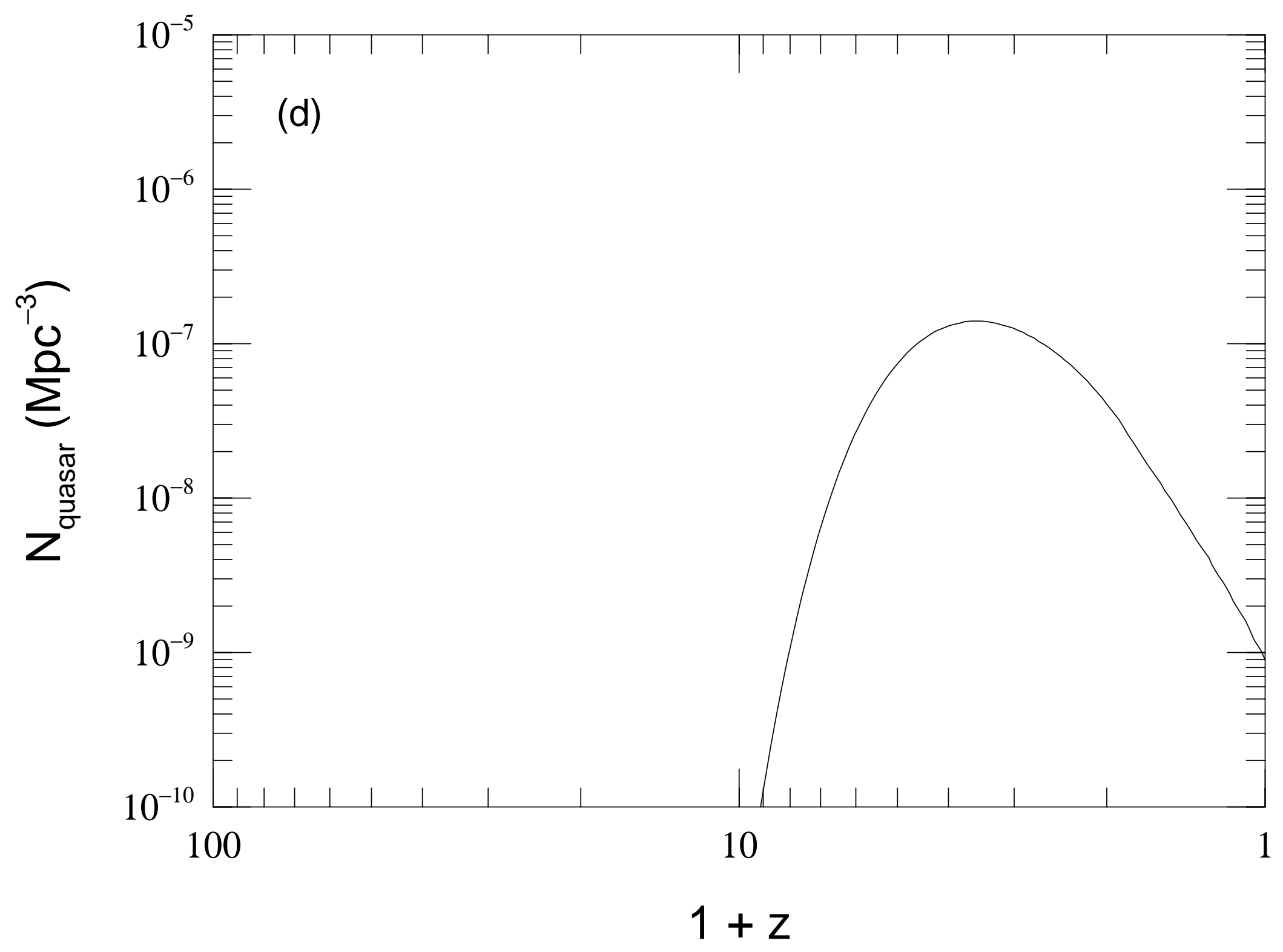

Fig.1(d) 


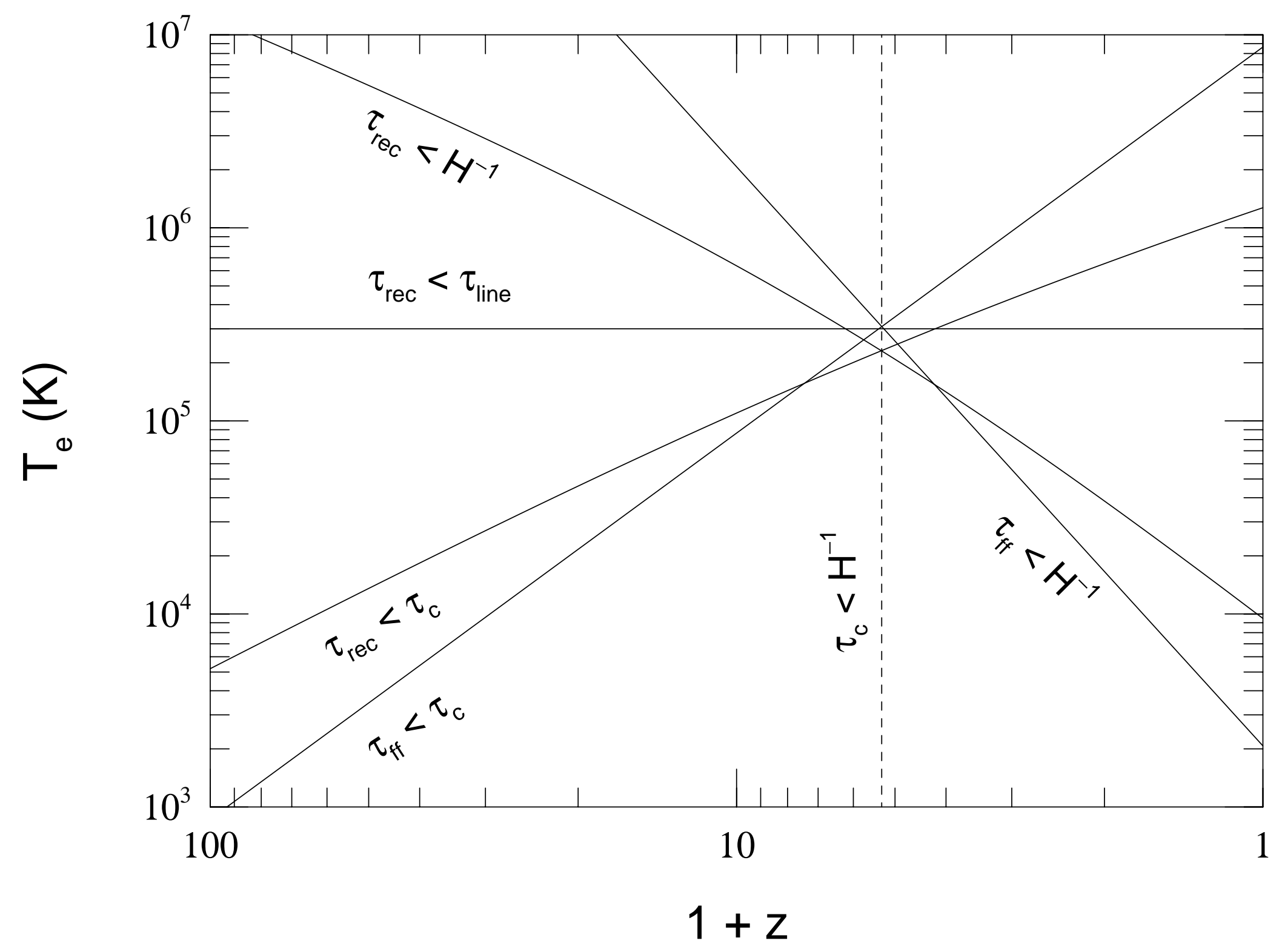

Fig.2 


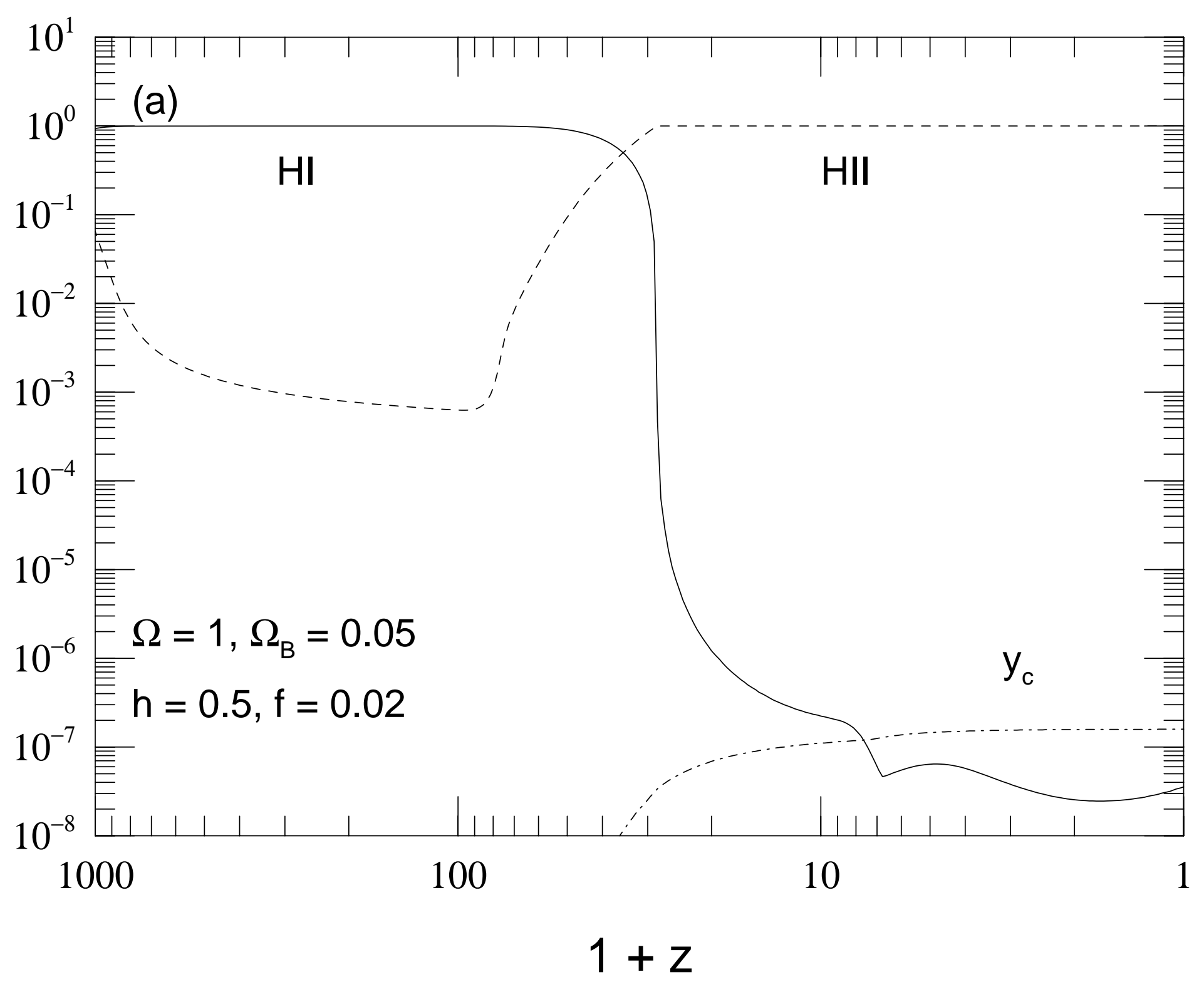

Fig.3(a) 


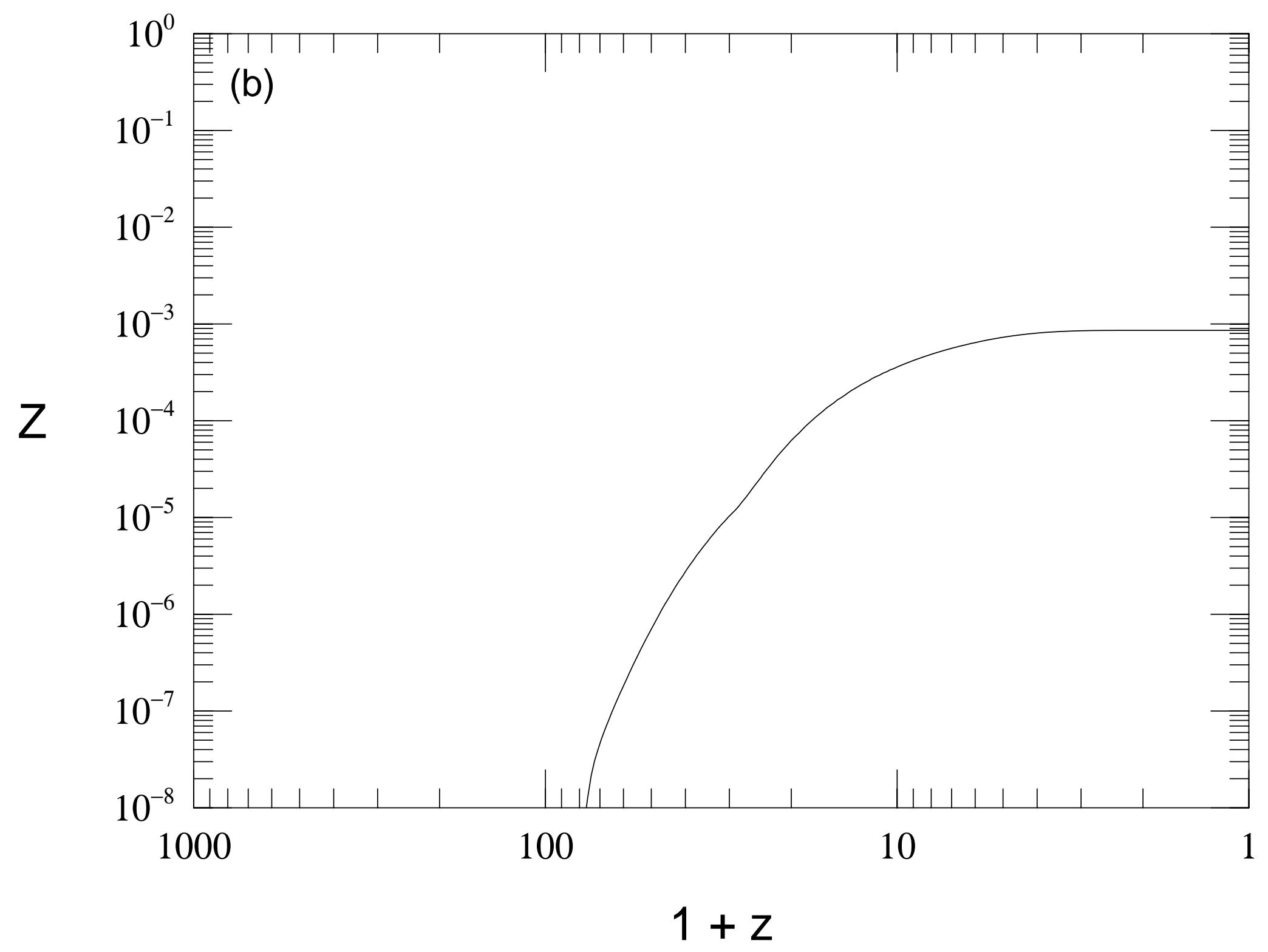

Fig.3(b) 


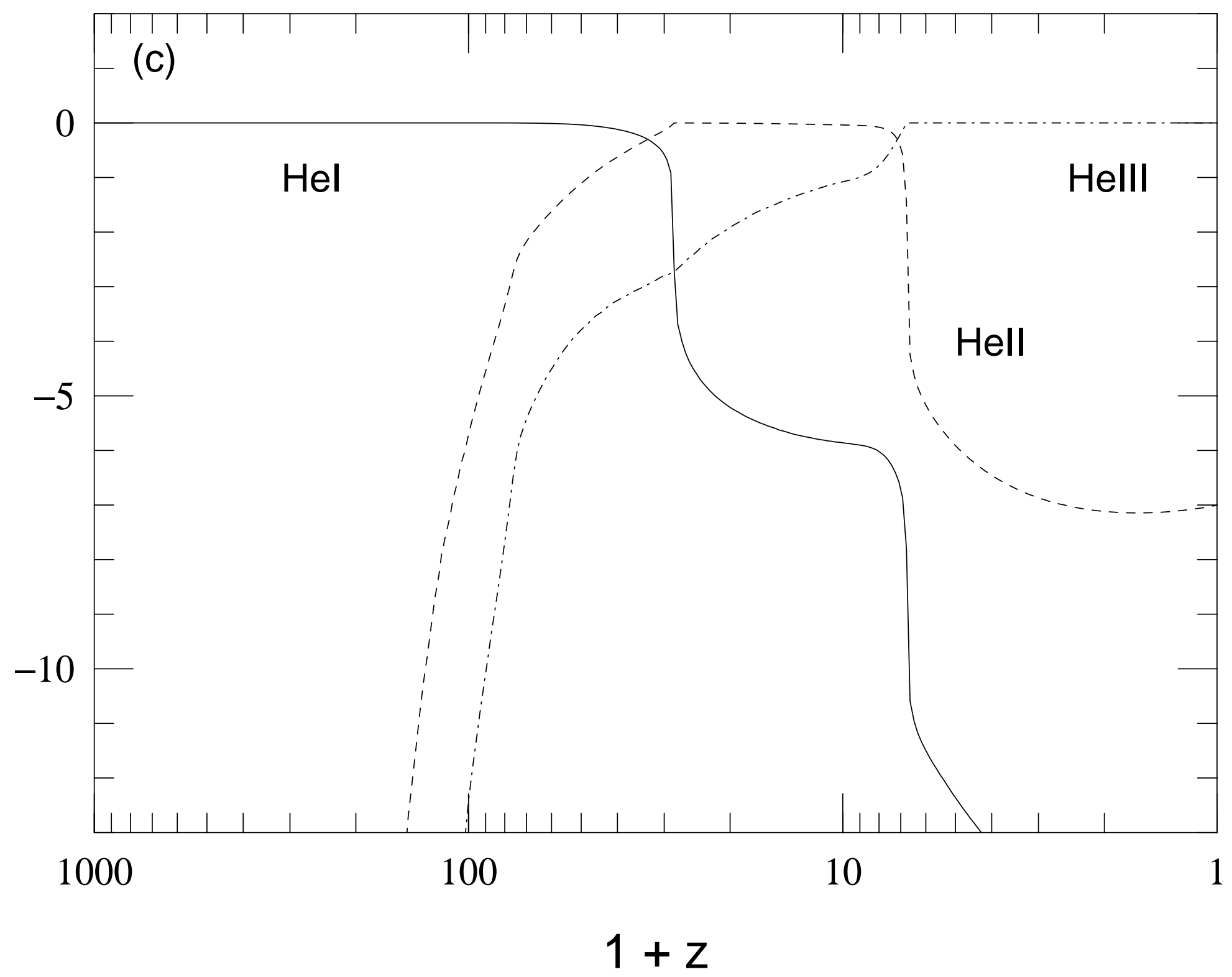

Fig.3(c) 


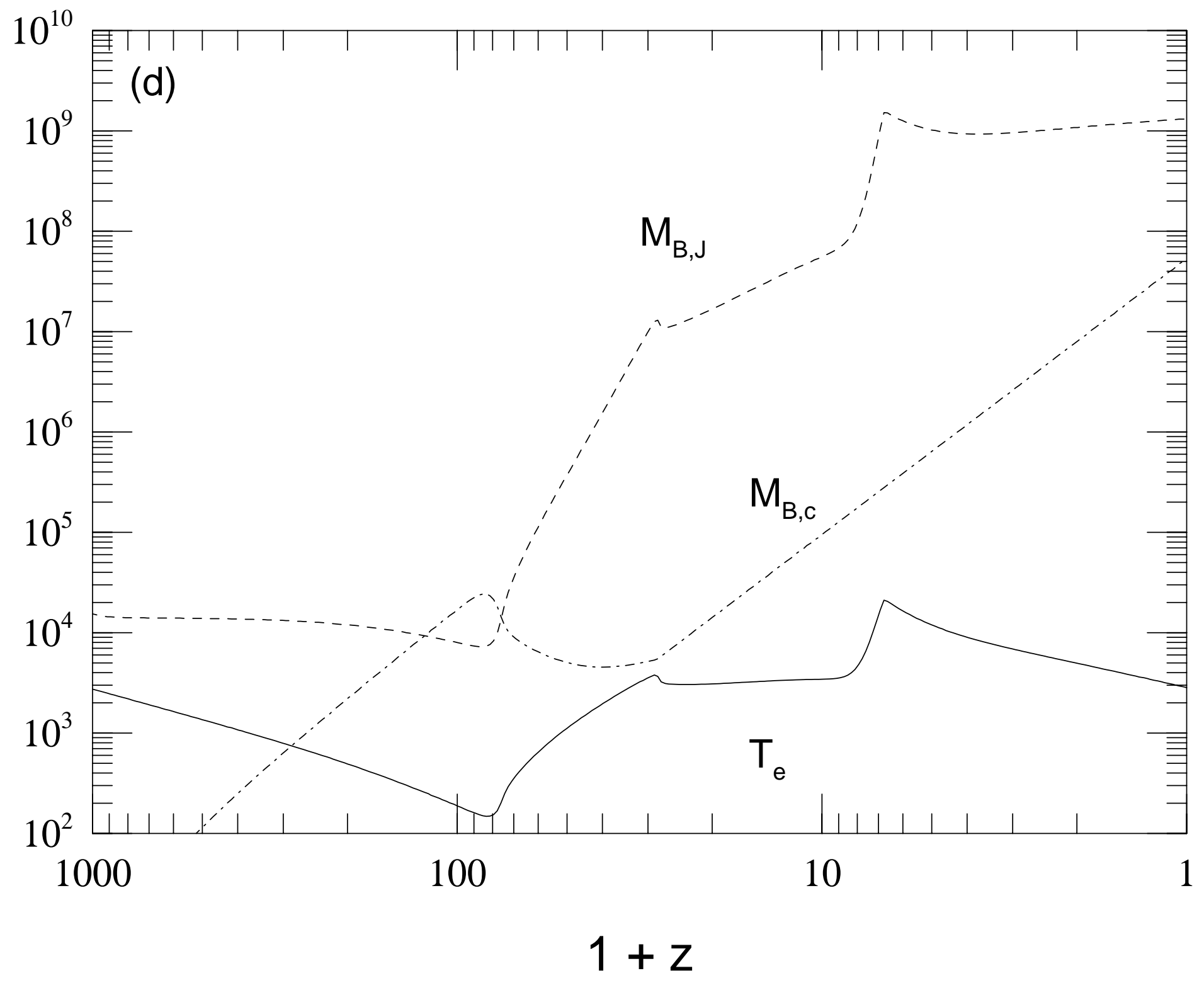

Fig.3(d) 


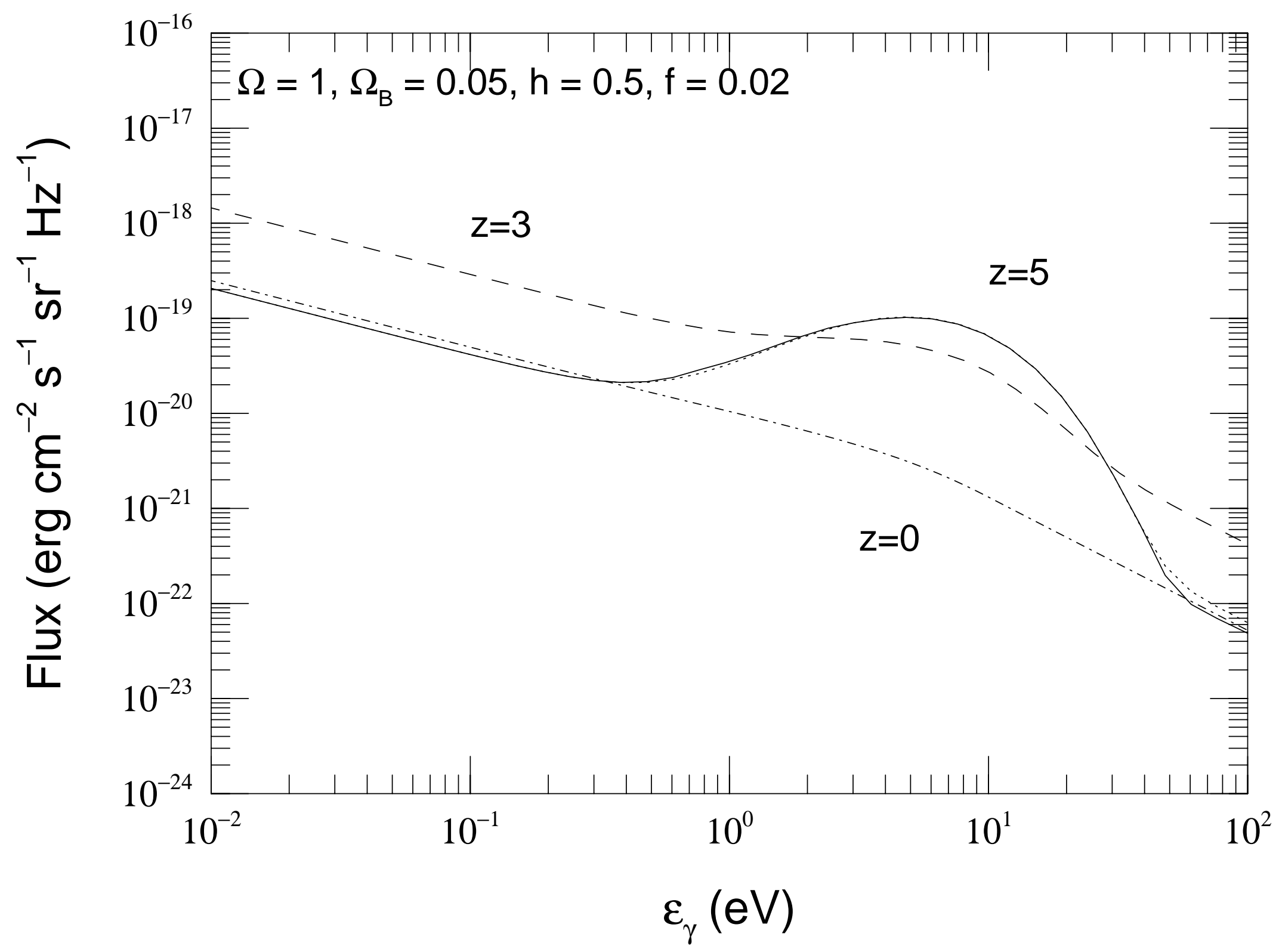

Fig.4 


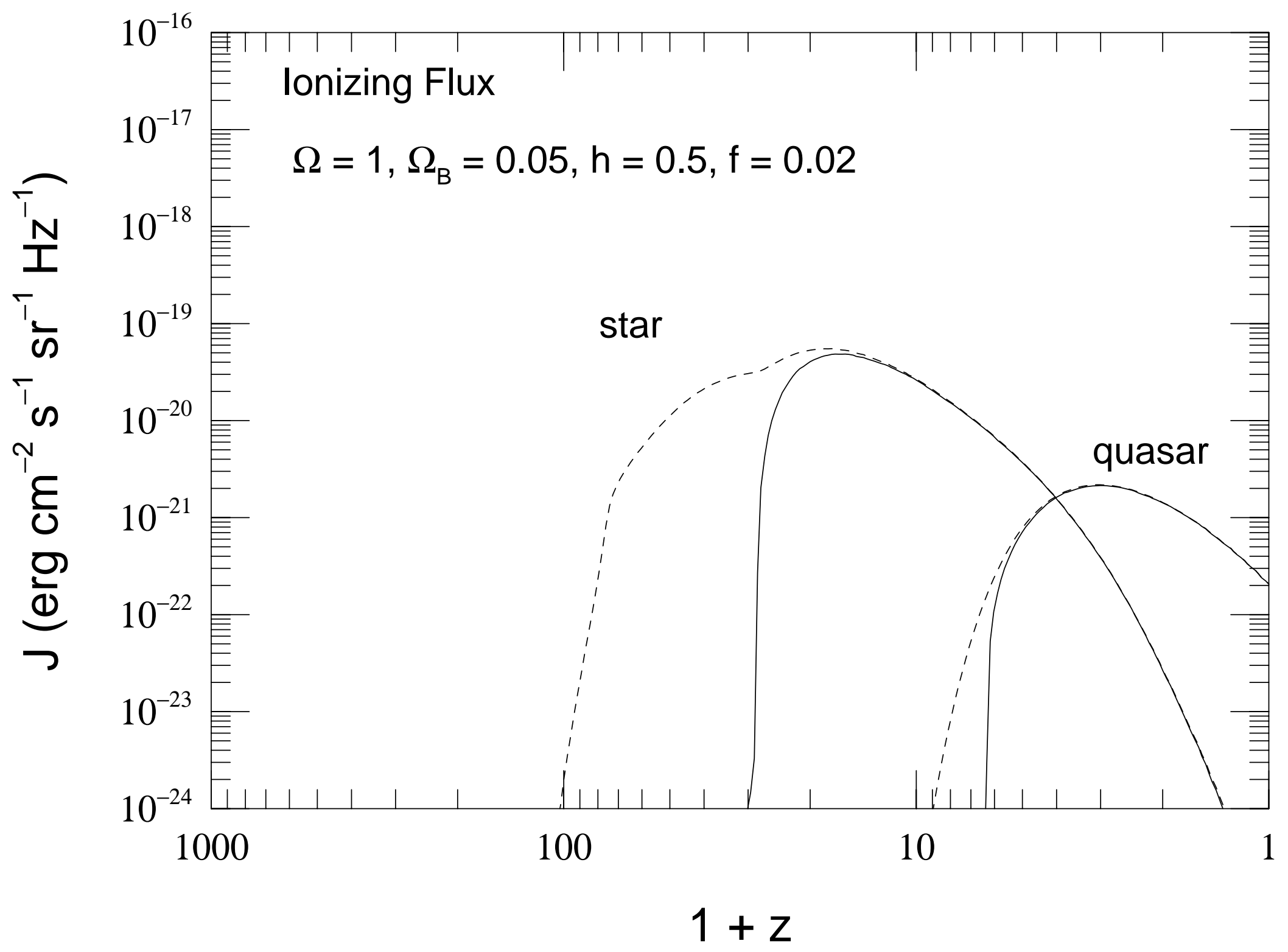

Fig.5 


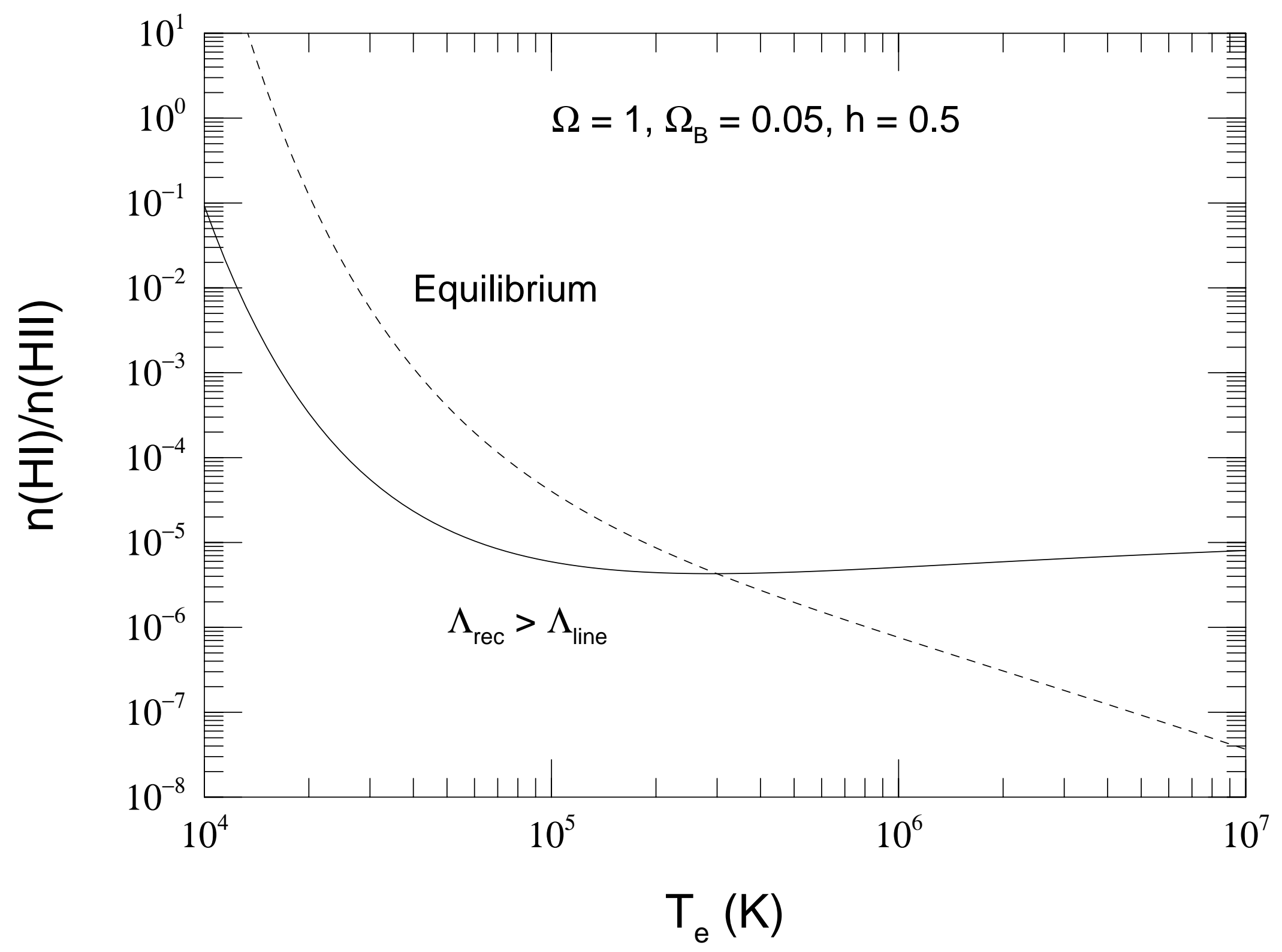

Fig.6 